\title{
PSYCHOLOGICAL RESPONSES TO NATURAL PATTERNS IN ARCHITECTURE
}

\author{
Alexander Coburn ${ }^{1}$, Omid Kardan², Hiroki Kotabe², Jason Steinberg ${ }^{2}$, Michael C. Hout ${ }^{3}$, Arryn \\ Robbins $^{3}$, Justin MacDonald ${ }^{3}$, Gregor Hayn-Leichsenring ${ }^{4}$, Marc G. Berman ${ }^{2}$ \\ ${ }^{1}$ University of Cambridge, ${ }^{2}$ University of Chicago, ${ }^{3} \mathrm{New}$ Mexico State University, ${ }^{4}$ University of Pennsylvania
}

\begin{abstract}
Exposure to natural environments, compared to built spaces, has been shown to confer important psychological benefits. Furthermore, people generally exhibit preferences for natural landscapes over man-made buildings. However, some forms of architectural design draw inspiration from natural systems and thus exhibit similar sensory qualities as nature itself. Can these natural features be measured, and are buildings which exhibit these features inherently preferred? Here, we examined whether subjective perceptions of naturalness in architectural scenes are driven by objective visual patterns, and we investigated whether natural patterns influence similarity evaluations and preference ratings of architectural scenes. Experiment 1 revealed that low-level spatial and color features of architectural scenes explained over half of the variance in scene naturalness ratings. Scaling and Contrast-related features were especially strong predictors of naturalness for both interior and exterior scenes. In Experiment 2, participants completed an image arrangement task and multidimensional scaling (MDS) analysis was performed on the data to determine the underlying aesthetic dimensions that drove scene similarity judgements. Naturalness ratings explained over half of the variance in MDS Dimension 1 weights, suggesting that people relied on latent perceptions of naturalness to evaluate the similarity of architectural scenes. In the final experiment, participants rated architectural scenes on aesthetic preference. Nature-like patterns of Scaling and Contrast significantly predicted preference ratings. Furthermore, the effects of Scaling and Contrast on preference were mediated by latent perceptions of naturalness, indicating that these architectural patterns are preferred because they evoke intuitive associations with sensory features of nature. Collectively, these studies suggest that people may be innately attuned to naturalistic features of the built environment, and that these features can be measured using computational methods of image analysis.
\end{abstract}

\section{Introduction}

The design of our physical surroundings - including landscapes and buildings - can have a meaningful impact on psychological states and wellbeing (Adams, 2014; Coburn, Vartanian, \& Chatterjee, 2017; Cooper \& Burton, 2014; Hartig, 2008; Joye, 2007b). The psychological benefits of naturalness, in particular, are widely documented in the environmental psychology literature (for a review, see Bowler, Buyung-Ali, Knight, \& Pullin, 2010). The sensory qualities of natural environments have been found to improve mood, attention, and 
cognitive functioning (Berman et al., 2012; Berman, Jonides, \& Kaplan, 2008; Berto, 2005; Bratman, Daily, Levy, \& Gross, 2015; Bratman, Hamilton, \& Daily, 2012; Bratman, Hamilton, Hahn, Daily, \& Gross, 2015; Kaplan, 1995), among other salubrious effects. Nature-like design features can also be found in certain built environments that exhibit visual patterns inspired by biological systems (Alexander, 2002; Goldberger, 1996; Joye, 2007b; Salingaros, 2003; Solomon, 2002). Researchers have proposed that organic patterns in architecture may be innately preferred over synthetic forms, and that exposure to naturalistic architectural spaces may confer similar psychological benefits as interacting with nature itself (Alexander, 2002; Joye, 2007b; Kellert, 2005; Salingaros, 2007). However, these ideas have received little experimental scrutiny to date (Joye, 2007b). Here, we examine whether subjective perceptions of naturalness in architecture are driven by objective visual patterns, and we investigate whether these nature-like patterns are robust predictors of similarity evaluations and preference ratings of architectural scenes. This work paves the way for future researchers to explore how naturalistic patterns in the built environment influence restoration and wellbeing.

\section{Psychological benefits of naturalness}

Previous research has shown that interacting with natural environments, compared to urban or built spaces, can confer important benefits for mental health. The salubrious effects of exposure to nature include improved mood (Barton \& Pretty, 2010; Bowler et al., 2010; Valtchanov, Barton, \& Ellard, 2010), reduced stress (Valtchanov et al., 2010; Villani \& Riva, 2011), improved concentration and working memory performance (Berman et al., 2012, 2008; Berto, 2005; Bratman, Daily, et al., 2015; Kaplan, 1995), higher self-esteem (Barton \& Pretty, 2010; Pretty, Peacock, Sellens, \& Griffin, 2005), increased feelings of energy and vitality (Ryan, Weinstein, Bernstein, \& Brown, 2010), and overall self-perceived health (Kardan, Gozdyra, et al., 2015). Views of nature have also been shown to reduce criminal behavior (Kuo \& Sullivan, 2001) and improve recovery from surgery (Ulrich, 1984). In fact, merely looking at images and virtual representations of natural landscapes can induce many of these benefits (Berman et al., 2008; Berto, 2005; Valtchanov et al., 2010; Valtchanov \& Ellard, 2015). 
Two complementary theories, the Biophilia Hypothesis $(\mathrm{BH})$ and Attention Restoration Theory $(A R T)$, help frame these empirical findings. ${ }^{1}$ The $\mathrm{BH}$ states that humans are innately drawn to the living and life-like forms often encountered in natural environments (Wilson, 1984; Wilson \& Kellert, 1995). Proponents of BH argue that people have a genetically-rooted need to seek contact with plants, animals, and natural places, which stems from our species' evolution in "biological - not artificial or manufactured - environment[s]" (Kellert, 2005, p. 123). The word biophilia, which means "love of life," emphasizes the emotional dimension of the human-nature connection. ART, on the other hand, focuses on the cognitive benefits people derive from interacting with nature. According to ART, softly fascinating sensory stimuli in nature engage our attention in an automatic, bottom-up manner, thereby replenishing the limited cognitive resources that govern top-down executive functions, such as concentrating on difficult tasks. Nature thereby "restores" attentional resources and facilitates better performance on demanding cognitive tasks (Kaplan, 1995; Kaplan \& Berman, 2010). Together, BH and ART offer complementary perspectives to explain why contact with nature might generate pleasurable and restorative psychological experiences.

In an effort to investigate these mechanisms more closely, Berman and colleagues (2014) identified specific visual patterns that may contribute to the cognitive and affective benefits of natural environments. Using image statistics, they quantified several low-level spatial and color patterns that reliably predicted whether outdoor environments were perceived as more natural or man-made. Common characteristics of natural-looking environments included high density of curved edges and high frequency of contrast changes distributed throughout the scene. In a follow-up study, Kardan and colleagues (2015) demonstrated that these naturalistic visual features were highly predictive of aesthetic preference ratings, suggesting that low-level patterns of nature may play an important role in generating aesthetic pleasure.

These findings have intriguing implications for architectural design and urban planning. Despite the salutary effects of nature, most people today spend upwards of $90 \%$ of their lives inside buildings (Evans \& McCoy, 1998). If the psychological benefits of natural environments are driven by measurable visual patterns, then could we optimize the visual properties of our built environment to create more pleasurable and restorative spaces for human inhabitation? Could the implementation of naturalistic patterns in architecture improve occupants' mood and cognitive functioning (Joye, 2007b; Kellert, 2005)? If so, then understanding how to

\footnotetext{
${ }^{1}$ Several other theories have also been proposed outlining potential mechanisms underlying the psychological benefits of exposure to nature, including Ulrich's stress reduction theory (Ulrich et al., 1991) and Joye's theory of processing fluency (Joye, Steg, Ünal, \& Pals, 2016).
} 
integrate the sensory characteristics of nature into the built environment could be a powerful tool for enhancing mental health on a large scale (Ibarra et al., 2017).

\section{Nature-like patterns in architecture}
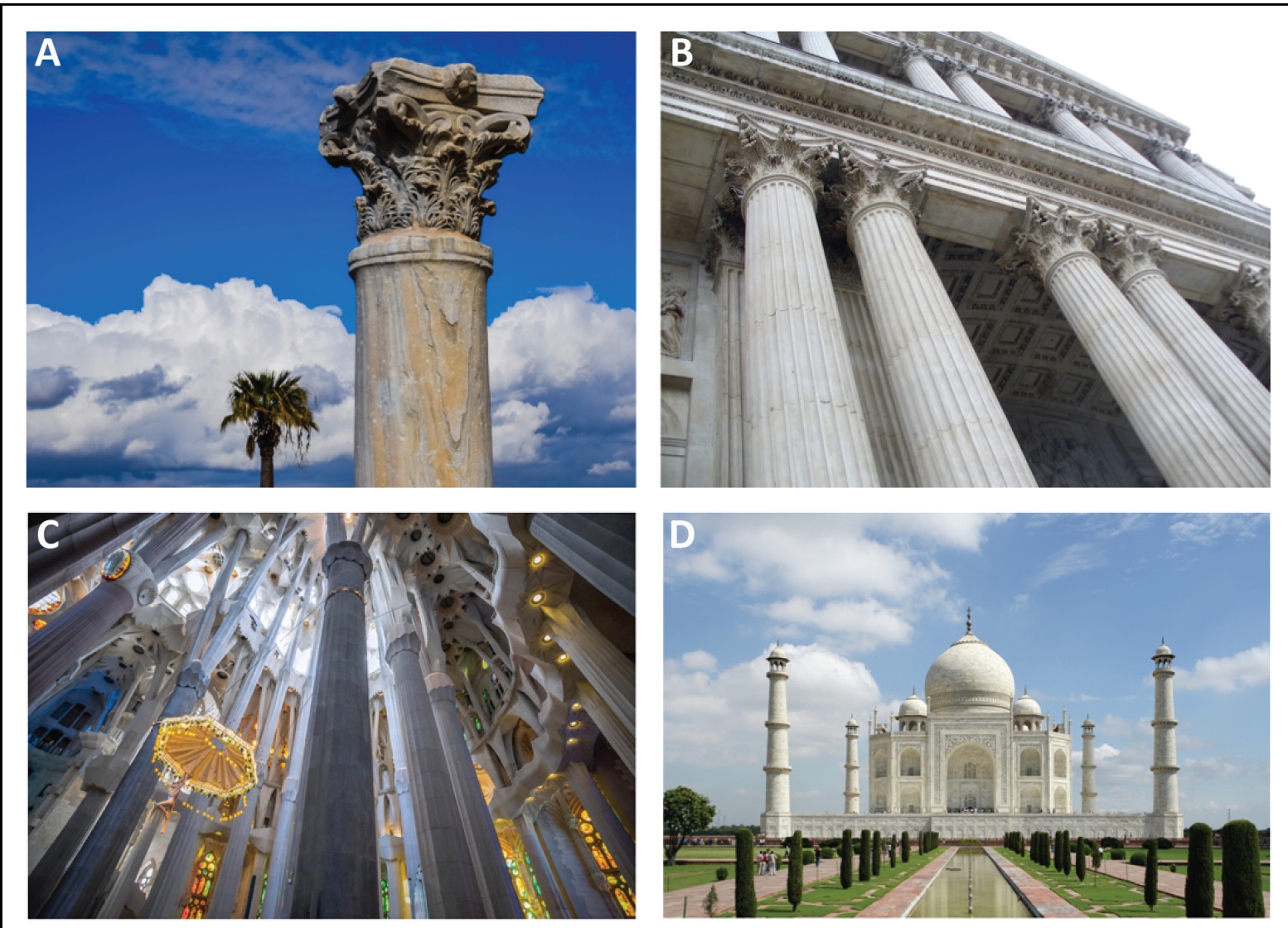

Figure 1: $(A, B)$ The Corinthian column's biologically-inspired design is evident in its tree-like structure and floral ornamentation (Tokkoro, 2018; Warder, 2008). (C) Sagrada Familia's structure resembles the branching canopies of trees (Joye, 2007b). (D) Taj Mahal (Forget, 2009) exhibits geometric patterns characteristic of biological systems, including local symmetries and self-similar shapes.

Although natural and manmade environments are often classified as categorically distinct types of space (Karmanov \& Hamel, 2008), many buildings across the globe exhibit naturelike characteristics. Naturalistic forms and patterns have long served as a fruitful source of inspiration for architects and builders around the world (Alexander, 2002; Joye, 2007b; Kellert, 2005; Ostwald, 2001; Salingaros, 2007). Kellert defines organic design as "building shapes and forms that directly, indirectly, and symbolically elicit a human affinity for natural features and processes" (Kellert, 2005, p. 128). Examples include literal imitations of animal and plant shapes in architectural ornamentation, engineering strategies that mimic the structural support mechanisms of biological organisms, and nature-like patterns of scaling and proportionality abstracted from natural systems (Figure 1). "These architectural elements," writes Kellert, "evoke sentiments that tap into our inherent responses to the patterns, movement, light, shape, and space encountered in nature" (Kellert, 2005, p. 159). 
Only a fraction of human construction, however, has arisen from the design model of nature (Kellert, 2003). Contemporary architecture often exhibits a different type of structural organization that is not rooted in nature's blueprints, but that is instead derived from intellectually-generated concepts like Euclidean geometry and the Cartesian coordinate system. Idealized shapes like rectangles, spheres, flat surfaces, and straight lines have increasingly dominated Western architecture since the Second World War (Figure 2), yet these inorganic forms are quite alien to the complex visual structures of living, biological systems (Aldersey-Williams, 2004; Kellert, 2005). ${ }^{2}$ Some researchers argue that the rise of Euclidean architecture in the $20^{\text {th }}$ century was driven by conscious efforts to create shocking new structures that stood out in stark contrast from nature (Alexander, 2002; Salingaros, 1998, 2007). Others contend that an increased emphasis on utilitarianism in building construction has moved architects away from using nature-based design models (Joye, 2007b), which are often perceived as incompatible with the economic incentives and production systems that drive contemporary development (Alexander, 2002). "Modern building is often dictated by efficiency and economic motives," writes Joye, "barely leaving room for symbolic and stylistic references to natural contents" (Joye, 2007b, p. 311).

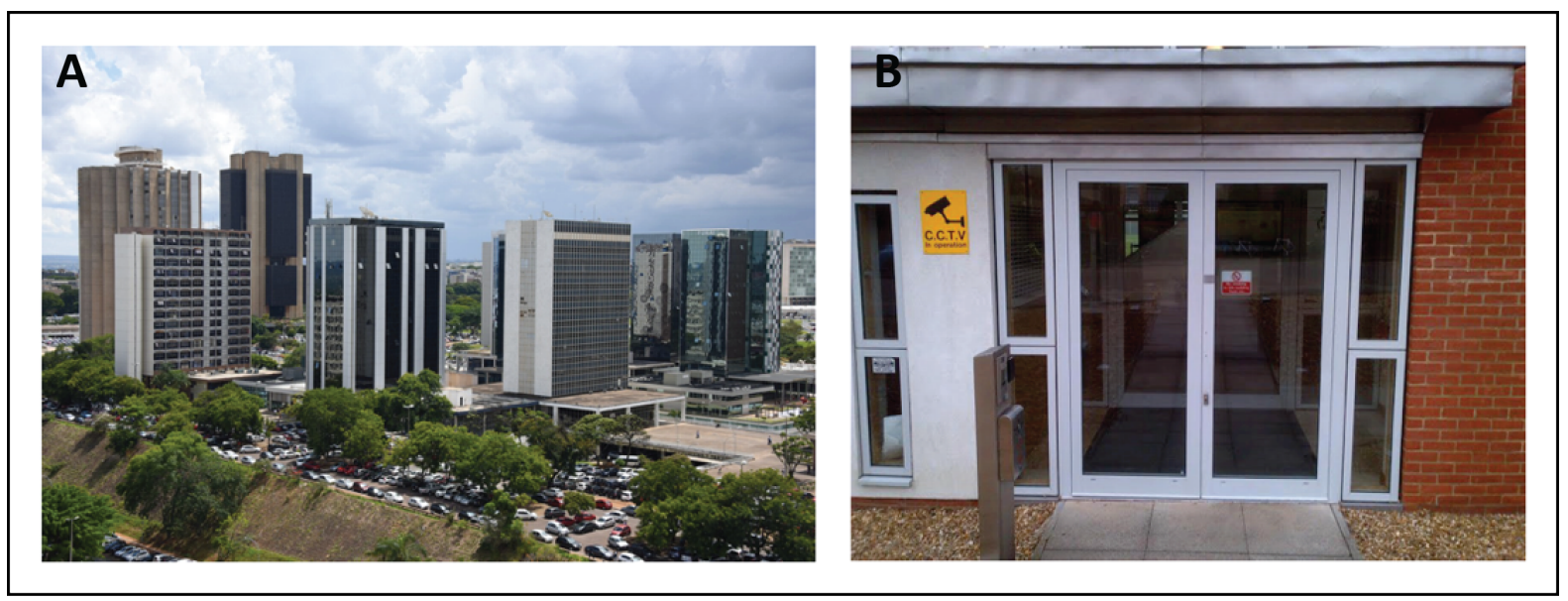

Figure 2: (A) Buildings in Brasilia, a city largely inspired by artificial geometric forms. (B) The design of a university dormitory in Cambridge, England is characterized by synthetic shapes.

How do natural vs. synthetic architectural forms impact human experience? Some scholars have asserted that humans are innately drawn to architectural forms that echo the organic qualities of nature (Alexander, 2002; Joye, 2006; Kellert, 2005; Ruskin, 1849; Salingaros, 2007). This idea bears a striking similarity to the aesthetic notions of philosopher Immanuel Kant, who proposed that all truly beautiful man-made objects (including buildings) look as if

\footnotetext{
${ }^{2}$ Kellert characterizes inorganic design as architecture that "reflects an excessive reliance on fabricated materials, artificial lighting, controlled climatic conditions, straight-line geometries, homogeneity of design, scales rarely if ever encountered in nature, [and] substitution of the synthetic for the natural" (Kellert, 2005, p. 133).
} 
they were created by nature. ${ }^{3}$ Other researchers have argued that exposure to nature-like architectural patterns may induce similar psychological benefits as interacting with nature itself (Alexander, 2002; Joye, 2007b; Kellert, 2003; Salingaros, 2007). Kellert, for instance, writes that organic architecture "enrich[es] the human body, mind, and spirit by fostering positive experiences of nature in the built environment" (Kellert, 2005, p. 5). However, very little empirical work to date has tested theoretical claims that naturalistic architecture is either innately preferred or restorative (Joye, 2007b).

A major challenge facing this line of research is the methodological difficulty of operationalizing naturalness in experiments. Reliable, objective measures of environmental naturalness are needed in order to investigate how this aesthetic quality influences psychological experiences. However, definitions of naturalness in the context of architecture are often ambiguous and inconsistent. For example, "natural architecture" can describe manmade structures that have nature-like characteristics, or it can refer to the presence of water, vegetation, and other natural features in and around buildings. ${ }^{4}$ However, neither of these definitions fully captures the overall degree of naturalness of an architectural space, which often depends on complex interactions among a variety of natural and built elements (Alexander, 2002; Kellert, 2005).

Instead of evaluating specific natural and built elements independently, our approach here investigates how visual patterns distributed throughout entire architectural scenes influence the perception of naturalness. In other words, we measure low-level sensory properties of whole environmental scenes rather than high-level semantic information derived from isolated objects within a scene (such as the amount of furniture in a room, or the number of trees surrounding a building). We predicted that low-level scene features would influence perceptions of naturalness independent of how much nature-related semantic content a scene contained. This prediction stems from previous evidence suggesting that semantic information can be carried by low-level scene features. For example, low-level edge and color

\footnotetext{
${ }^{3}$ Kant observed that natural as well as man-made objects can be beautiful. In his view, nature is beautiful if it looks man-made, and man-made objects are beautiful if they look natural (Kant, 2001). This vice versa 'as if' is the key to Kant's concept of beauty. He proposed that the creation of beautiful objects requires inborn talent. During the act of creation, the so-called genius does not follow formalistic rules, but is instead guided by his or her natural intuition. Therefore, according to Kant, man-made beauty is closely associated with the quality of naturalness.

${ }^{4}$ Biomorphic design models architectural forms after biological structures, whereas biophilic design focuses on incorporating water, vegetation, and sunlight into the built environment.
} 
patterns have been found to convey semantic information about order and naturalness (Kotabe, Kardan, \& Berman, 2016a). ${ }^{5}$

We integrate two complementary strategies, one empirically-motivated and the other theory-driven, for identifying natural features of architectural design. The first strategy uses image statistics to identify low-level spatial and color properties of architectural scenes that drive subjective naturalness ratings. This approach builds on previous experiments showing that low-level visual features strongly predict perceptions of naturalness in outdoor landscapes (Berman et al., 2014; Ibarra et al., 2017; Kardan, Demiralp, et al., 2015) and that naturalistic spatial and color features play a role in driving scene preference ratings (Kardan, Demiralp, et al., 2015). We predict that these low-level scene features, when integrated into architectural design, will evoke associations with the environmental quality of naturalness.

The second strategy is derived from the concept of living structure (Alexander, 2002). According to this theory, architecture exhibits naturalistic, or living, aesthetic qualities when the process by which it is constructed resembles the adaptive, structure-preserving processes of biological growth (Alexander, 2004). ${ }^{6}$ When people build as nature does, Alexander argues, buildings develop nature-like geometric patterns in their structure, which are summarized as fifteen patterns of living structure (Figure 3). While some of these patterns are difficult to quantify, others lend themselves to empirical measurement. Intriguingly, two of the patterns - Levels of Scale and Contrast - align closely with the low-level visual features that Berman et al. (2014) and Kardan et al. (2015) identified in their experimental work as predictable characteristics of natural environments. These two patterns are qualitatively described in Box 1. In the subsequent experiments, we quantify them using image statistics and investigate their relationship with subjective naturalness and preference ratings of architectural spaces.

Figure 3: Fifteen patterns of living structure (Alexander, 2002)

\begin{tabular}{|l|l|l|}
\hline Levels of Scale & Strong Centers & Boundaries \\
\hline Alternating Repetition & Positive Space & Good Shape \\
\hline Local Symmetries & Deep Interlock & Contrast \\
\hline Gradients & Roughness & Echoes \\
\hline The Void & Simplicity and Inner Calm & Not-Separateness \\
\hline
\end{tabular}

\footnotetext{
${ }^{5}$ This prediction is also motivated by the idea that representations of the natural world in architecture depend as much on intuitive recognition of visual patterns abstracted from natural systems as on explicit depictions of biological forms (Alexander, 2002; Joye, 2007b; Kellert, 2005; Salingaros, 1998).
}

${ }^{6}$ For detailed examples of adaptive construction processes, see Alexander (2004) The process of creating life. 


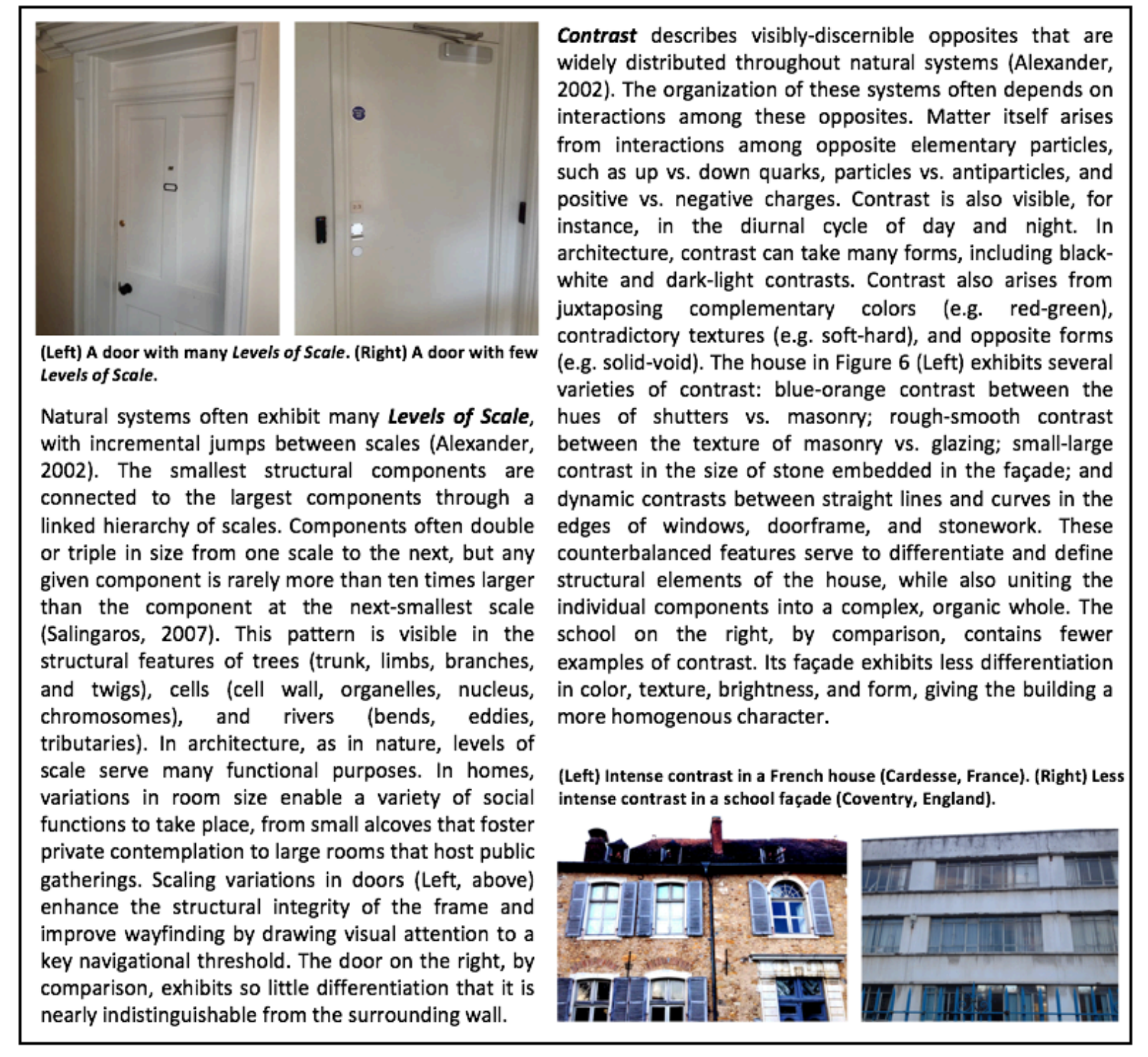

Box 1: Descriptions of Levels of Scale and Contrast, two proposed patterns of living structure (Alexander, 2002; Salingaros, 2007).

\section{Overview of experiments}

The purpose of this research was to investigate whether subjective perceptions of naturalness are driven by objective spatial and color features of buildings and to determine whether these naturalistic design features influence similarity ratings and aesthetic preferences of architectural spaces. We addressed these questions in three experiments. In Experiment 1, we collected subjective naturalness ratings of interior and exterior architectural images and regressed these ratings on nine low-level image features. We predicted that low-level spatial and color patterns would explain a significant proportion of the variance in naturalness ratings, and that buildings exhibiting more Levels of Scale and greater visual Contrast would be perceived as more natural. In Experiment 2, we asked participants to judge the similarity of diverse architectural images using an image arrangement task. We applied multidimensional scaling analysis (MDS) to these similarity data to identify the underlying 
aesthetic dimensions that drove participants' image arrangement decisions (Berman et al., 2014; Hout, Papesh, \& Goldinger, 2013; Shepard, 1980), predicting that the way in which participants intuitively organized images would be influenced by latent perceptions of naturalness. We tested this prediction by regressing dimension weights from the MDS analysis on subjective naturalness ratings collected in the first experiment. In Experiment 3, we collected preference ratings for the architectural images and tested the hypothesis that preferences would be strongly predicted by the naturalistic patterns quantified in Experiment 1. We predicted that nature-like Scaling and Contrast features, specifically, would explain a significant proportion of the variance in preference ratings, and that the effects of Scaling and Contrast on aesthetic preference would be mediated by latent perceptions of naturalness in architectural scenes.

\section{Experiment 1: Identifying nature-like patterns in architecture}

In this experiment, we set out to determine whether subjective perceptions of naturalness are driven by objective low-level features of architectural scenes. We predicted that low-level scene features would significantly predict naturalness ratings, and that architectural scenes exhibiting greater Levels of Scale and greater visual Contrast would be perceived as more natural. We also carried out a comparative analysis of interiors and exteriors to examine how our nine spatial and color features correlated with naturalness ratings across our two image sets. Finally, we investigated the role that high-level semantic content played in predicting perceptions of naturalness for our images.

\section{Methods}

\section{Participants}

100 American adults ( 55 Women, 45 men) were recruited for this experiment from Amazon's Mechanical Turk (MTurk) to rate images of architectural spaces on their perceived level of naturalness. Sample size was determined by our goal of obtaining approximately 50 naturalness ratings per image (Kotabe, Kardan, \& Berman, 2016b, 2017). Half of participants (Group 1) were assigned to rate images of interior spaces $(n=50)$, and the other half (Group 2 ) were assigned to rate images of exterior spaces $(n=50)$. Ages ranged from 21 to 65 years $(M=34.6, S D=9.6)$. Data was excluded from 8 participants who gave the same naturalness rating for 10 or more consecutive stimuli at least once during their individual trial. This response pattern suggested that they were likely clicking through the images and not attending to the assigned task. All participants were compensated $\$ 1.00$ for their participation and the experiment took approximately 10 minutes to complete. Informed 
consent was obtained through the Institutional Review Board (IRB) of the University of Chicago.

\section{Materials}

Two sets of stimuli were used in all three experiments of this study: 120 images of architectural interiors and 120 images of architectural exteriors. Interior photographs were chosen from a variety of online public domain collections of architectural images. Exterior photographs were taken from the Street View interface of Google Earth and were restricted to head-on shots of buildings taken at a distance of 20-30 feet from the façade. Within each 120-image stimulus set, twenty diverse examples of architectural spaces were chosen for each of six building types (commercial, educational, government, residential, medical, and religious). ${ }^{7}$ This selection process strengthened the external validity of our study by exposing participants to a variety of architectural spaces representative of diverse building types one would encounter in the real world. It also enabled us to control for the potentially confounding effect of building function on architectural evaluations and ensured that both stimulus sets contained the same distribution of images across all six functional categories, thus facilitating more reliable comparison between interior and exterior results. The amount of vegetation (i.e. plants, trees) depicted in the image sets was intentionally minimized in the selection process in order to reduce the confounding effects of non-architectural natural features on subjective naturalness ratings of buildings. Images were normalized to 4:3 widthto-height ratios with dimensions of $1175^{*} 881$ pixels for exteriors and $1000 * 750$ pixels for interiors to ensure dimensional consistency across each image set. The images can be downloaded here: https://github.com/alexcoburn11/Natural-Buildings-Images.

\footnotetext{
${ }^{7}$ Although this is not an exhaustive list of possible building functions, it fulfilled our purpose of diversifying our stimuli and balancing both image sets across a range of functional categories.
} 


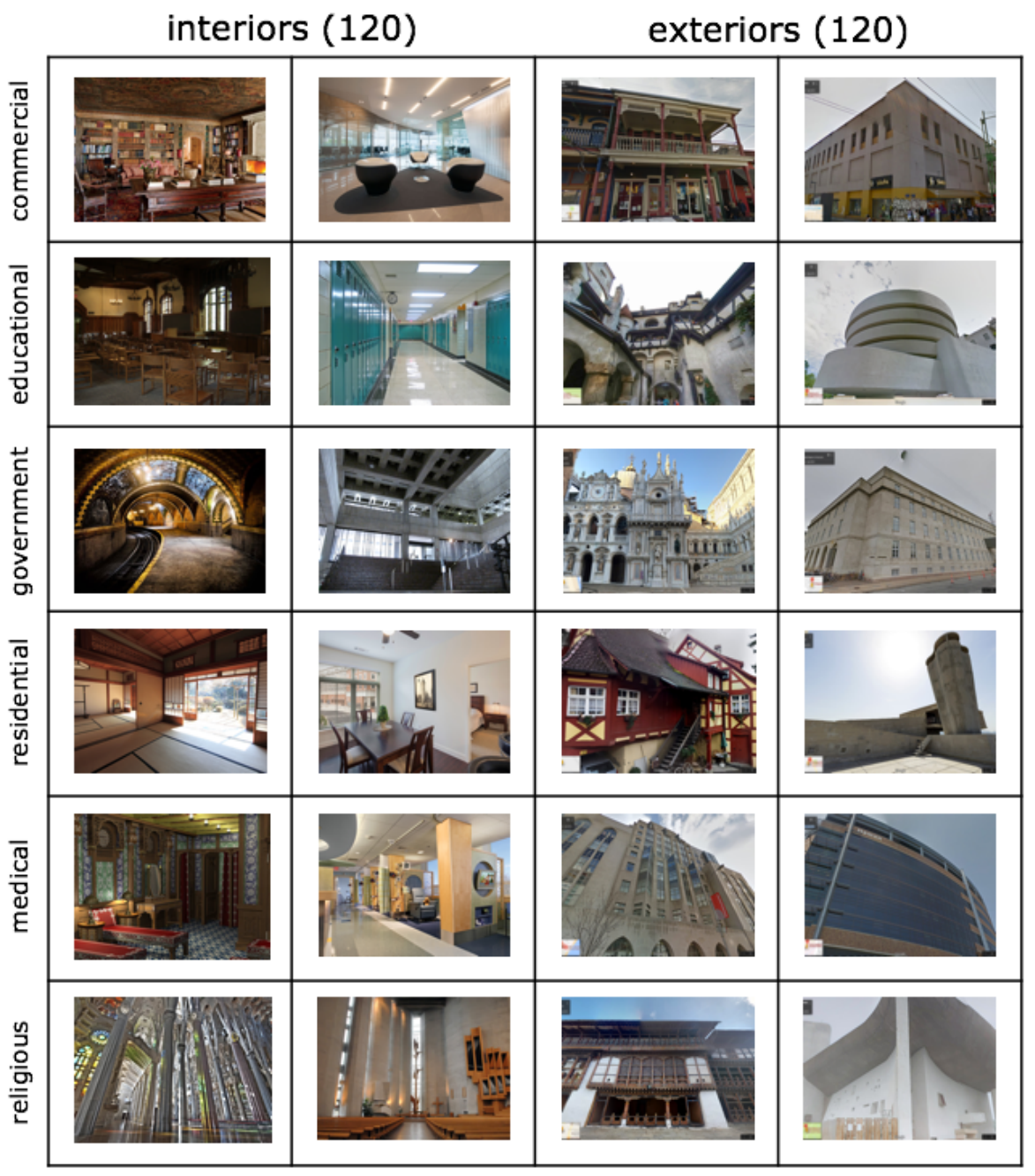

\section{Procedure}

Participants rated images using the online interface of Qualtrics survey software. Participants in Group $1(n=50)$ were shown the 120-image set of interior architectural spaces in a randomized order and were asked to rate each image in response to the prompt, "How artificial or natural does this building interior look to you?" Answer choices were presented on a standard 7-point Likert scale, with 1 indicating "very artificial" and 7 indicating "very natural." Participants were given unlimited time to rate each image. The same procedure was followed for participants in Group $2(n=50)$, except that they were asked to rate the set of 120 images of architectural exteriors rather than interior spaces.

\section{Quantifying spatial and color properties of architecture}

We measured three spatial properties and six color properties of each scene in order to estimate the degree to which naturalness ratings could be statistically explained by these objective visual features. There are many possible ways in which visual properties of images can be analyzed. Here, we measured a set of visual features that were assessed in two 
previous studies investigating the low-level visual correlates of naturalness in outdoor spaces (Berman et al., 2014; Kardan, Demiralp, et al., 2015). We focused on these particular measures for three reasons: 1) they have straightforward interpretations; 2) they can be easily manipulated in visual stimuli by researchers and in built environments by architectural designers; and 3) they are theoretically relevant to the patterns of natural structure described in the introduction (Alexander, 2002).

\section{Spatial Properties}

Three spatial features of the images were calculated in this study. 1) Edge Density is a measure of how many straight and curved edges are in an image. This statistic was calculated using methods described in (Berman et al., 2014; Kardan, Demiralp, et al., 2015). The term "edges" describes points of discontinuity in brightness in an image that represent object boundaries and small-scale details of texture. Edge maps were calculated for each scene using MATLAB's built-in Canny edge detection algorithm. Edge density was calculated from these edge maps as the sum of total edge pixel values ( 0 for non-edge pixels, 0.5 for faint edges, 1 for strong edges) divided by the total number of pixels in the image.

2) Fractal Dimension, as measured in our study, captures the visual complexity and scaling differentiation of the edge maps of our architectural images. Unlike smooth Euclidean shapes, fractals are fractured shapes consisting of self-similar patterns that occur on many scales of magnification, "building scale-invariant shapes of immense complexity" (Taylor et al., 2005, p. 91). Whereas a smooth Euclidean curve has a fractal dimension close to 1 , a densely convoluted line that approximates the appearance of a two-dimensional surface has a fractal dimension closer to 2 . Fractal dimension was calculated here by creating edge maps of the architectural images using the built-in Canny edge detection function in MATLAB. We then calculated the fractal dimension of each image's edge map using the "boxcount" algorithm in MATLAB (Moisy, 2008), which applies the box-counting technique documented in (Taylor et al., 2005). ${ }^{8}$

3) Entropy is a statistical measure of randomness in a scene that is calculated using the scene's intensity histogram. A histogram of a gray scale image shows the distribution of intensity values of all the pixels that comprise the image. For an 8-bit grayscale image, each pixel could have an intensity value of $0-255$. If the histogram of such an image has 256 bins (i.e., one bin

\footnotetext{
${ }^{8}$ The box-counting method counts the number $(\mathrm{N})$ of identical 2-dimensional boxes of size $(\mathrm{R})$ needed to cover all edges (i.e., all nonzero pixels) in the image. This analysis is repeated for boxes with a range of square sizes. Each decrease in box size $(R)$ represents an increase in magnification. Box sizes vary by powers of two, i.e., $R=1,2,4 \ldots 2 P$ where $\mathrm{P}$ is the smallest integer such that $2 \mathrm{P}$ is smaller than the total image size. For fractal images, $\mathrm{N}$ scales according to the equation $N=R-D$, where $D$ is the fractal dimension of the image and $1<D<2$.
} 
for each possible intensity value), then the probability value of the $n$th bin of the histogram $\left(p_{n}\right)$ is calculated as the number of pixels with an intensity value of $n-1$ divided by the total number of pixels in the image. Entropy was calculated using the following equation:

$$
\text { Entropy }=-\sum_{n=1}^{256}\left(p_{n} * \log _{2} p_{n}\right)
$$

This equation (Kardan, Demiralp, et al., 2015) gives an estimate of the average information content of an image. High entropy values indicate that all possible intensity values in an image occur with the same probability (i.e., the intensity histogram represents a uniform distribution), suggesting that there is a high degree of randomness in the distribution of intensity values. Conversely, low entropy values indicate that the distribution of intensity values throughout a scene are non-uniform (i.e., non-random), suggesting more redundancy in informational content of the image.

\section{Color Properties}

Six color properties of the images were calculated based on the standard hue-saturationvalue (HSV) model using the built-in functions of MATLAB image processing toolbox (MATLAB and Image Processing Toolbox Release 2016b, The MathWorks, Inc., Natick, MA, USA). Hue is a measure of the average color appearance of an image (i.e., the dominant color wavelength of the scene). Saturation describes the intensity or purity of colors in a scene (i.e., the ratio of the dominant wavelength to all other wavelengths in a color). Brightness captures the average luminance or value of an image. Standard deviations of these three color properties were also measured to quantify the diversity of hue (sdHue), the diversity of saturation (sdSat), and the diversity of brightness (sdBright) in each image. Calculations of all image statistics were normalized to the size of each image by dividing by the total number of pixels in the image. After all nine low-level visual features were calculated, values were standardized for each statistic by calculating Z-scores within each image set. 


\section{Quantifying Levels of Scale and Contrast}

We used several of these image statistics to operationalize two of Alexander's proposed patterns of natural structure, Levels of Scale and Contrast (Alexander, 2002). When buildings exhibit many Levels of Scale, the smallest structural details are connected to the largest visible components through a linked hierarchy of scales. Our Fractal Dimension measure is a close approximation of this pattern, since it measures the degree to which edge patterns in an image repeat at many scales of magnification. Architectural scenes with low Fractal Dimension values (approaching 1) are likely to depict smooth, sparse surfaces with little scaling differentiation and with large jumps between scales. Images with high Fractal Dimension values (approaching 2 ) $^{9}$ generally depict more intricate, detailed structures (Taylor et al., 2005), with more scales present and smaller jumps in between scales. A high degree of scaling differentiation is also generally associated with a greater density of small-scale details, whereas less differentiated (i.e., minimalist) spaces typically contain less detail. Edge Density, which captures the amount of detailed edges in a scene, is therefore another close proxy for Alexander's Levels of Scale pattern.

The Contrast pattern was evaluated using three of our low-level color features: sdHue, sdSat, and sdBright. These three statistics serve as useful proxies for three distinct forms of visual contrast, as they directly measure the diversity of color (sdHue), the diversity of color intensity (sdSat), and the diversity of brightness (sdBright) distributed throughout an architectural scene. These are by no means the only types of contrast that can exist in a building (see Box 1 for more examples of contrast). However, they are straightforward and quantifiable examples of visual contrast features. We predicted that all five of these statistical proxies of Levels of Scale and Contrast would correlate positively with naturalness ratings for both interior and exterior image sets.

\footnotetext{
${ }^{9}$ This is because images are 2-Dimensional.
} 


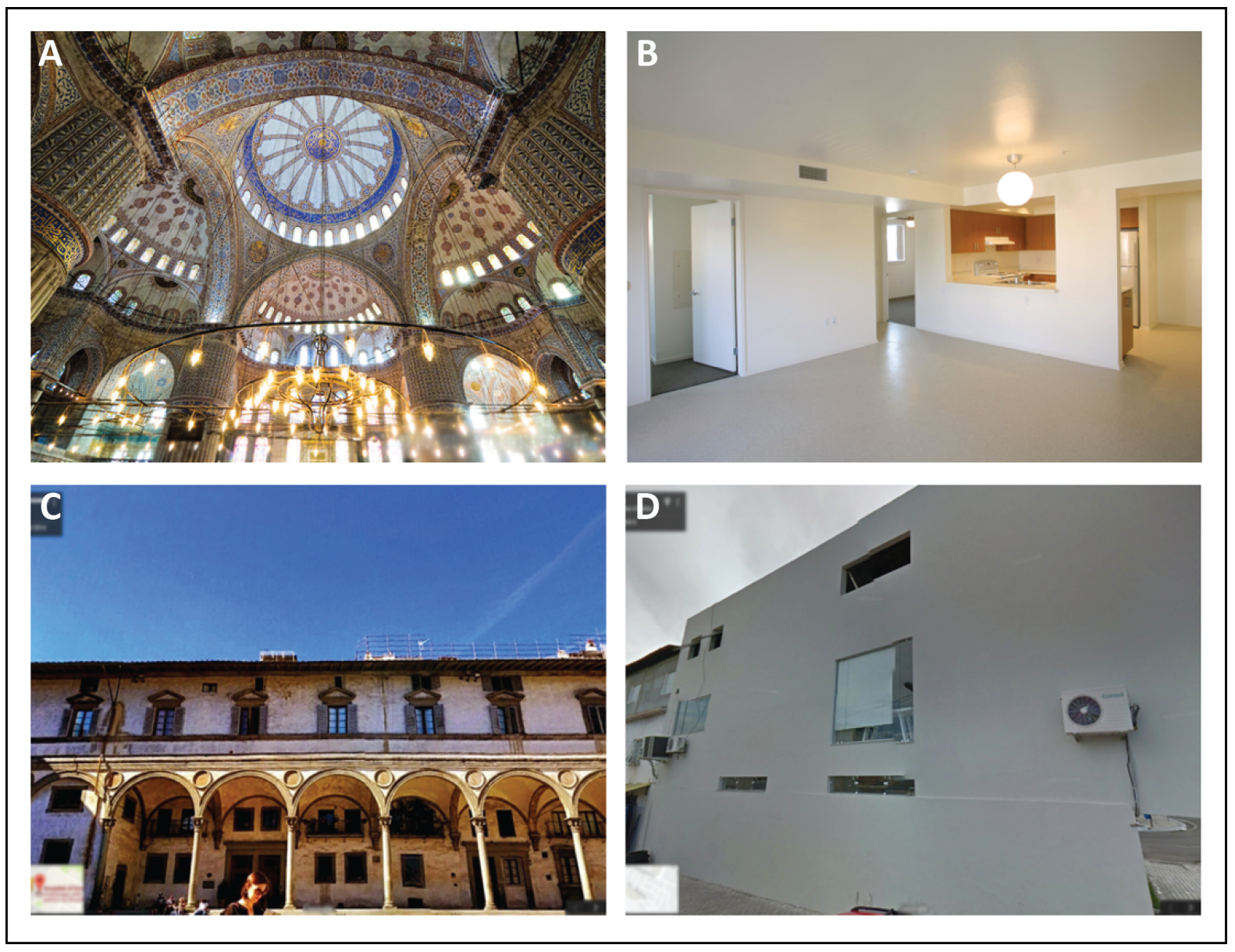

Figure 4: (A) Interior with high values of edge density $(Z=+2.56)$ and fractal dimension $(Z=+1.77)$. (B) Interior with low values of edge density $(Z=-2.01)$ and fractal dimension $(Z=-2.73)$. (C) Exterior with high values of sdHue $(Z=+0.83)$, sdSat $(Z=+2.20)$, and sdBright $(Z=+2.02)$. (D) Exterior with low values of sdHue $(Z=-0.79)$, sdSat $(Z=-1.67)$, and sdBright $(Z=-1.70)$.

\section{Checking for redundant measures}

Next, correlation matrices were computed for our interior and exterior image sets to check for multicollinearity in our low-level visual features (Figure 5). Edge Density and Fractal Dimension were highly correlated in both interior $(r=0.96)$ and exterior $(r=0.91)$ images, indicating that these were essentially redundant measures. We combined these variables by calculating the average values of Edge Density and Fractal Dimension for each image. We labeled this new combined variable Scaling, since both of the measures that are theoretically linked to Alexander's Levels of Scale pattern. 


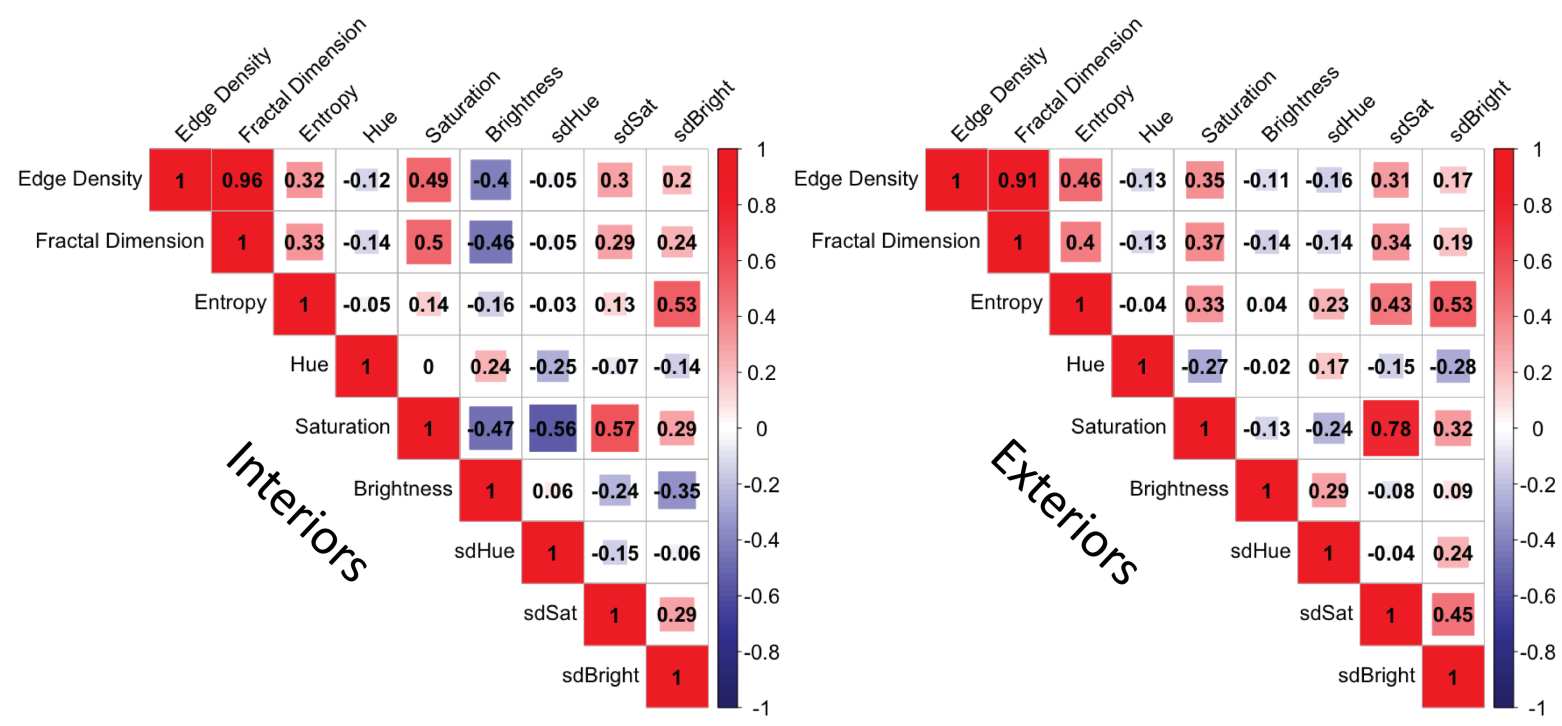

Figure 5: Correlation matrices of image statistics for Interiors (Left) and Exteriors (Right)

\section{Quantifying explicit nature}

In order to control for the natural vegetation content of our scenes, we minimized the presence of explicit natural content in the stimuli, but also measured the number of pixels in each scene depicting any remaining natural vegetation (e.g. grass, bushes, trees, flowerpots) using the Quick Selection tool in Adobe Photoshop, and then divided this value by the total pixel area of the scene. The resulting variable, which we called Explicit Nature, represented the proportion of image area occupied by vegetation in each architectural scene. This variable was added to regression models to control for the presence of vegetation in the architectural scenes.

\section{Statistical analysis}

Analyses were conducted at the image level by calculating average naturalness ratings for each image across all participants. Linear multiple regression models were constructed to examine the relationship between low-level visual features and mean naturalness ratings of interior and exterior image sets. In a second pair of analyses, we regressed naturalness ratings on Explicit Nature scores to determine the effect of high-level semantic content on subjective perceptions of naturalness.

\section{Results}

\section{Spatial and color features predict perceptions of naturalness}

We constructed a linear multiple regression model for the data from Group 1 participants (the group who was shown the images of interiors) by regressing mean naturalness ratings of 
the interior images on eight low-level spatial and color features. The Explicit Nature variable was added to the regression model to control for the amount of vegetation present in the scenes. The majority of the variance in mean naturalness ratings was collectively explained by these nine visual features $\left[R^{2}\right.$ adj $\left.\left.=0.66, F(9,110)=26.40, P<.001\right)\right]$. Low-level visual features independently explained over half $(54 \%)$ of the variance in naturalness ratings when controlling for Explicit Nature. ${ }^{10}$ We performed the same analysis on the data from Group 2 participants (the group who was shown the images of exteriors). The nine visual features of exterior images also significantly predicted their mean naturalness ratings $\left[R^{2}\right.$ adj $=0.52, F(9$, $110)=15.04, P<.001)$ ]. When controlling for Explicit Nature, low-level visual features independently explained $42 \%$ of the variance in naturalness ratings for exterior scenes. The results of these two regressions build on previous work showing that low-level visual features significantly predict the perception of naturalness in outdoor environmental scenes (Berman et al., 2014; Kardan, Demiralp, et al., 2015), many of which contained little or no built structure. Here, these past findings are extended to images of the built environment.

As shown in Tables 1 and 2, higher values of Scaling, sdSat, and sdBright significantly predicted higher naturalness scores for both interior and exterior architectural spaces. Additionally, Brightness correlated negatively with naturalness for interior scenes, although this effect was barely significant $(P=0.045)$. Since images with high Scaling values are indicative of greater scaling differentiation in architectural design, the strong positive correlation found for this measure supports the hypothesis that incremental scaling is associated with the perception of naturalness in architectural scenes, whereas images of buildings with more abrupt changes in scale are more likely to be perceived as artificial-looking. The Scaling measure independently explained $31.7 \%$ and $15.8 \%$ of variance in naturalness ratings for interior and exterior scenes, respectively.

\footnotetext{
${ }^{10}$ The relatively low amount of variance explained by Explicit Nature was likely influenced by the fact that scenes with minimal plants and vegetation were intentionally chosen in the image selection process in order to minimize the confounding effects of non-architectural references to nature on perceptions of naturalness.
} 
Table 1: Regression of naturalness ratings vs. image features (interiors)

\begin{tabular}{lllllll}
\hline Visual Feature & Estimate & SE & $\boldsymbol{\beta}_{\text {ST }}$ & $t$ value & $\boldsymbol{P}$ value & $\boldsymbol{n}_{\boldsymbol{p}}{ }^{2}$ \\
\hline Scaling** & 0.452 & 0.063 & 0.507 & 7.149 & $<.001$ & 0.317 \\
Entropy & -0.102 & 0.059 & -0.116 & -1.723 & 0.088 & 0.026 \\
Hue & -0.044 & 0.051 & -0.050 & -0.855 & 0.395 & 0.007 \\
Saturation & 0.118 & 0.089 & 0.134 & 1.327 & 0.187 & 0.016 \\
Brightness* & -0.122 & 0.060 & -0.139 & -2.031 & 0.045 & 0.036 \\
sdHue & -0.106 & 0.066 & -0.120 & -1.614 & 0.109 & 0.023 \\
sdSat* & 0.136 & 0.061 & 0.154 & 2.241 & 0.027 & 0.044 \\
sdBright** & 0.173 & 0.061 & 0.196 & 2.817 & 0.006 & 0.067 \\
Explicit Nature** & 7.174 & 2.517 & 0.157 & 2.851 & 0.005 & 0.069 \\
\hline
\end{tabular}

$R_{\text {adj }}^{2}=0.66, F(9,110)=26.40, P<.001$

Table 2: Regression of naturalness ratings vs. image features (exteriors)

\begin{tabular}{lllllll}
\hline Visual Feature & Estimate & SE & $\boldsymbol{\beta}_{\text {ST }}$ & t value & $\boldsymbol{P}$ value & $\boldsymbol{n}_{\boldsymbol{p}}{ }^{2}$ \\
\hline Scaling** & 0.289 & 0.064 & 0.352 & 4.543 & $<.001$ & 0.158 \\
Entropy & -0.107 & 0.071 & -0.133 & -1.518 & 0.132 & 0.021 \\
Hue & -0.016 & 0.058 & -0.019 & -0.268 & 0.789 & 0.001 \\
Saturation & -0.106 & 0.090 & -0.132 & -1.176 & 0.242 & 0.012 \\
Brightness & 0.066 & 0.054 & 0.082 & 1.222 & 0.225 & 0.013 \\
sdHue & 0.027 & 0.061 & 0.033 & 0.437 & 0.663 & 0.002 \\
sdSat*** & 0.362 & 0.090 & 0.451 & 4.029 & $<.001$ & 0.129 \\
sdBright** & 0.227 & 0.071 & 0.282 & 3.191 & 0.002 & 0.085 \\
Explicit Nature** & 1.716 & 0.631 & 0.189 & 2.719 & 0.008 & 0.063 \\
\hline$R^{2}$
\end{tabular}

$R^{2}{ }_{a d j}=0.52, F(9,110)=15.04, P<.001$

Furthermore, two of the three features of color contrast - sdSat and sdBright - correlated significantly with naturalness in both image sets, thus supporting the hypothesis that greater visual contrast is positively associated with the perception of naturalness in architectural scenes. This effect, however, was limited to saturation and brightness-related contrast patterns, as hue diversity (sdHue) was not a significant predictor of naturalness in either image set. The contrast-related measures independently explained $13.4 \%$ and $21.6 \%$ of variance in naturalness ratings for interior and exterior scenes, respectively.

In summary, these results show consistent relationships between low-level visual features, especially Scaling and Contrast-related patterns, and subjective perceptions of naturalness for both interior and exterior architectural scenes. Since both regression models controlled for the effect of vegetation on naturalness ratings, the results imply that Scaling and Contrastrelated patterns visible in the buildings themselves, rather than in the trees and plants surrounding them, were driving perceptions of naturalness for these two image sets. These findings are consistent with the hypothesis that two of Alexander's proposed patterns of natural structure - Levels of Scale and Contrast - are positively associated with perceptions of naturalness in architectural design. 


\section{Experiment 2:}

\section{Does naturalness of buildings influence similarity perceptions?}

In this experiment, we set out to investigate whether latent perceptions of natural patterns in architectural scenes influence intuitive judgments of scene similarity. First, naïve participants assessed the similarity of diverse architectural images in an image arrangement task referred to as the spatial arrangement method (SpAM; Hout, Goldinger, \& Ferguson, 2013). We then applied multidimensional scaling analysis (MDS) to these similarity data to identify the underlying aesthetic dimensions that drove participants' grouping decisions (Berman et al., 2014; Hout et al., 2015; Hout, Papesh, et al., 2013; Shepard, 1980), predicting that latent perceptions of naturalness would strongly predict image grouping behavior. We tested this prediction by regressing dimension weights from the MDS analysis on subjective naturalness ratings collected in the first experiment. Finally, we investigated whether naturelike Scaling and Contrast features significantly predicted perceptions of scene similarity.

\section{Methods}

\section{Participants}

One hundred and sixty-seven participants, 81 from the University of Chicago, and 86 from New Mexico State University, took part in this study. All participants provided written informed consent. As compensation for their participation, University of Chicago participants were offered the choice of $\$ 10$ cash payment or partial course credit towards psychology courses. Participants from New Mexico State University were compensated with partial course credit towards introductory psychology courses. Each participant completed the spatial arrangement task on one of four sets of stimuli (selection was counterbalanced across participants), which are referred to as Exteriors A (43 participants), Exteriors B (41 participants), Interiors A (41 participants), and Interiors B (42 participants).

\section{Materials}

The stimuli used in this study were the same 240 images of interior $(n=120)$ and exterior $(n=120)$ architectural scenes used in Experiment 1. Here, however, each 120-image set was divided evenly into two sets of 66 interior scenes and two sets of 66 exterior scenes, with 12 images overlapping between the two sets for each scene type to check across sample stability of MDS dimensions. All photographs were JPG format, resized to $360 \times 270$ pixels so that multiple of them could be presented simultaneously (see procedure). Stimulus presentation was controlled by E-Prime vs 2.0 (Psychological Software Tools, 2012), presented on monitors that were $62.5 \mathrm{~cm} \times 32.5 \mathrm{~cm}$, at a resolution of $3840 \times 2160$. 


\section{Procedure}

On each trial, 20 different pictures were shown to the participant, randomly arranged in four rows of five items (evenly spaced along the $x$ - and $y$-axes). Participants were instructed to use the mouse to drag-and-drop the images in order to arrange them according to the participant's perceived similarity of each pair (with closer in space denoting proportionately greater similarity and vice versa; see Hout \& Goldinger, 2016; Hout, Goldinger, et al., 2013). Participants were allowed as much time as they needed to arrange each set of pictures, and clicked on a small (100x100 pixels) image of a stop sign (placed in the bottom-most right corner of the display) to indicate that they were done arranging the stimuli. After clicking on the stop sign, they were provided with a prompt asking them if they were done arranging the stimuli, if they needed more time, or if they would like to start over. This prompt ensured that trials were not ended prematurely in the event that the stop sign was clicked by mistake. If the participant indicated that they would like to start over, all images were returned to their original starting configuration. When the participant indicated that they were done, the program recorded the $\mathrm{x}$ - and $\mathrm{y}$-coordinates for each image, and the Euclidean distance between each pair (for 20 stimuli, there are 190 pairwise distances). This procedure was performed 18 times, with different sets of pictures on each trial, ensuring that each image was paired with every other image at least once. Therefore, each participant provided a complete similarity matrix for the set of 66 scenes (i.e., 2,145 pairwise distances).

We controlled the selection of images on each trial by employing a stimulus selection algorithm (MacDonald, Hout, \& Schmidt, in preparation; algorithm code is available at http://justinmacdonald.net/publications) that attempts to minimize the number of trials necessary to ensure that all possible pairings of pictures were included in at least one trial ${ }^{11}$. The algorithm chose 20 -item subsets of our full set of 66 stimuli such that every pair of items was presented together on at least one trial, and the total number of trials was minimized. Because a list of subsets in which each pair of items is presented exactly once does not exist (for details, see Horsley, 2017), some items were paired with others on more than one trial. This leads to multiple observations per "cell," so in such instances, we took the average of the multiple distances to use as the similarity rating for that pair. To balance out these redundancies, across participants, images were randomly assigned to numerical identifiers in the algorithmic set. This ensured that each participant saw each pair of pictures together at least once, but that different participants were presented with different redundant pairings.

\footnotetext{
11 This is a special case of the set cover problem in combinatorics (Vazirani, 2001): a block of $k$ items is sampled repeatedly from a larger set of $n$ items. How many blocks of items are necessary so that all possible $t$-sized subsets appear together within a block at least once $(n>k>t)$ ? In our experiment, $n=66$ items, $k=20$ items per trial, and $t=$ 2 , indicating that we wanted all 66-choose-2 item pairs to appear in a 20 -item trial at least once.
} 


\section{Determining dimensionality of data}

Similarity data for each of the four sets of stimuli were subjected to multidimensional scaling via the PROXSCAL scaling algorithm (Busing, Commandeur, Heiser, Bandilla, \& Faulbaum, 1997) implemented in SPSS software. Metric MDS was performed, as Euclidean distances on the computer monitor are of ratio scale. To determine the appropriate dimensionality in which to scale our data, Scree plots were created, plotting the model's stress against the number of dimensions used to locate the points in space. Stress functions measure the agreement between the modelled distances provided by the MDS output and the raw input proximities (i.e., the raw Euclidean distances between pairs). Lower stress values indicate better model fit. Scree plots are often used to determine dimensionality by having the analyst look for an "elbow" in the plot; that is, the point at which stress no longer decreases substantially with increased dimensionality. Pronounced elbows are not always present on Scree plots, however, and as you can see from our results, stress continued to decrease across many dimensions, nearing zero at six dimensions for each of our four stimulus sets. It is also worth noting that the stress functions are remarkably similar for all four sets of images. When large sets of stimuli are employed in MDS, it is less deleterious to overestimate the dimensionality of the space than it is to underestimate dimensionality (Hout, Cunningham, Robbins, \& MacDonald, 2018). As such, we scaled each of our stimulus sets in six dimensions. 


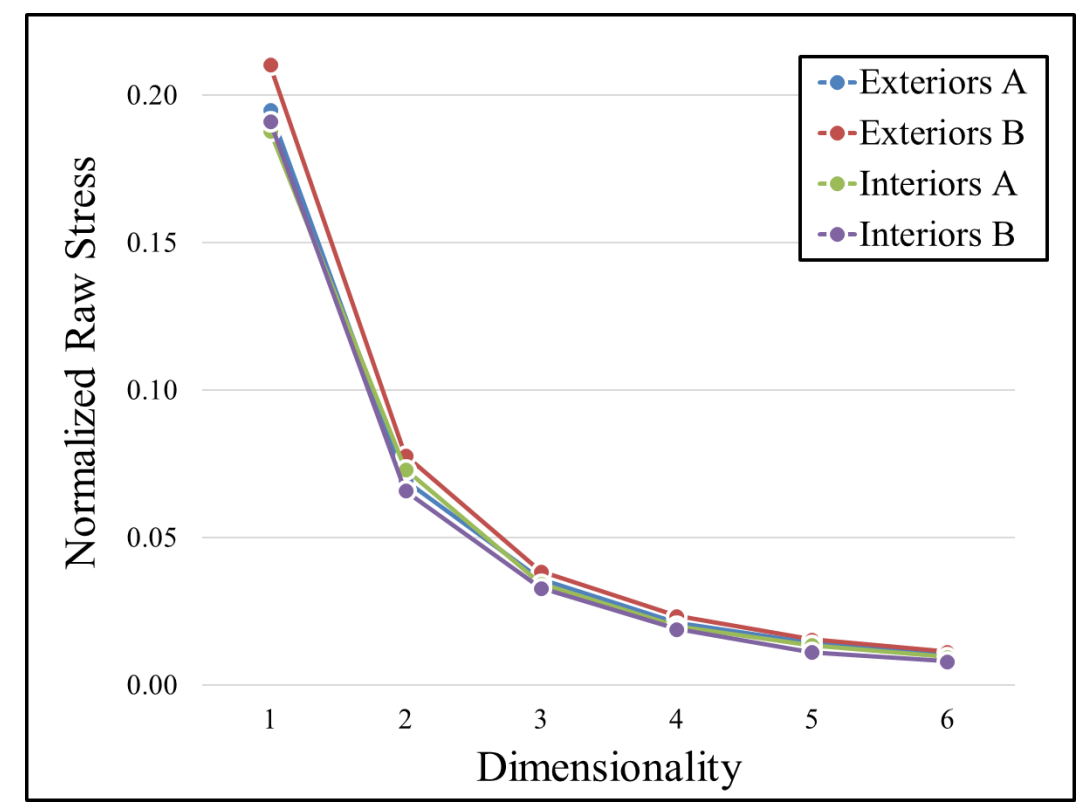

Figure 6: Scree plots, showing normalized raw stress (for each of the four stimulus sets) plotted against the number of dimensions used in the MDS analysis.

\section{Results}

\section{MDS analysis}

The results of the MDS analysis on the four sets of images are displayed in Figure 7, Figure 11, Figure 12, Figure 13, respectively. In those visualizations, the architectural images are superimposed on the MDS plot according to their weights on Dimension 1 (X-axis) and Dimension 2 (Y-axis). As mentioned, the data were scaled in six dimensions to yield the most appropriate overall spatial organization. However, the analysis here focused on the weights of the first two dimensions, as those dimensions explained the most variance in image similarity. At first glance, Dimension 1 appeared to code for the naturalness of the architectural scenes, with scenes depicting more naturalistic buildings having higher weights on Dimension 1, and with images depicting more artificial-looking buildings having lower weights on Dimension 1. Dimension 2 was more difficult to interpret. This pattern emerged across all four sets of images. 


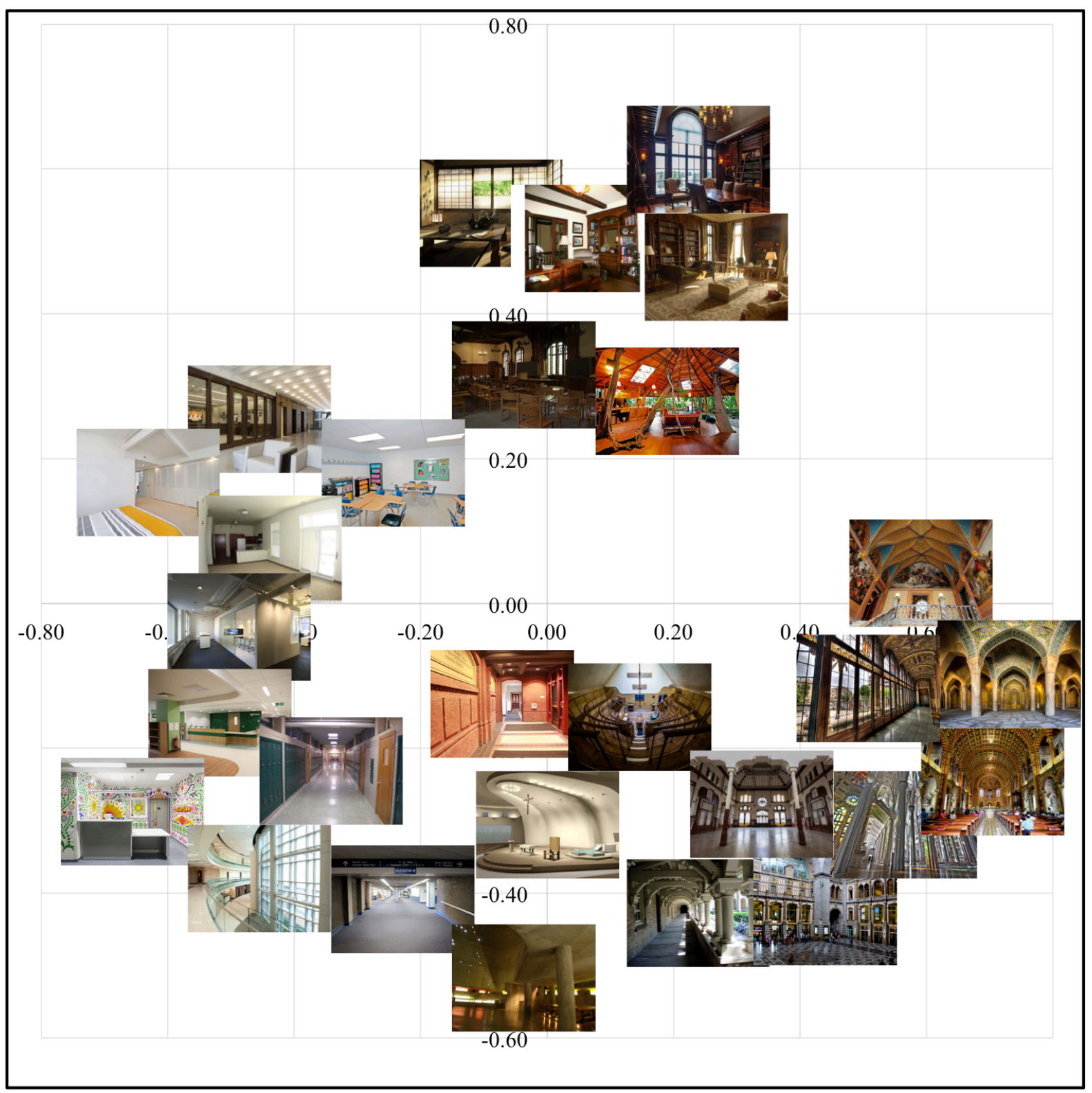

Figure 7: Plotted results of MDS dimension 1 (X-axis) and Dimension 2 (Y-axis) for the first image set (Interiors A). Pictures are superimposed based on their weights on Dimensions 1 and 2 . A subset of the 66 images is plotted in order to make the graph more readable.

\section{Naturalness predicts similarity ratings}

To test whether Dimension 1 from the MDS analysis was coding for latent perceptions of naturalness, we constructed linear regression models of images' Dimension 1 weights as a function of their mean naturalness ratings (collected in Experiment 1). Subjective naturalness ratings significantly predicted Dimension 1 weights across all four sets of images. Naturalness ratings explained over half of the variance in Dimension 1 weights for Interiors $\mathrm{A}\left[R^{2}\right.$ adj $=0.57$, $F(1,64)=88.02, P<.001]$ and Interiors $B\left[R^{2}\right.$ adj $\left.=0.53, F(1,64)=73.87, P<.001\right]$ and over twothirds of the variance in Dimension 1 weights for Exteriors $A\left[R^{2}\right.$ adj $=0.68, F(1,64)=141.1, P<$ 
$.001]$ and Exteriors $B\left[R^{2}\right.$ adj $\left.=0.73, F(1,64)=179.6, P<.001\right]$. Scatterplots showing correlations between naturalness ratings and Dimension 1 weights are shown in Figure 8.

Dimension 1 weights vs. Naturalness ratings (Interiors A)

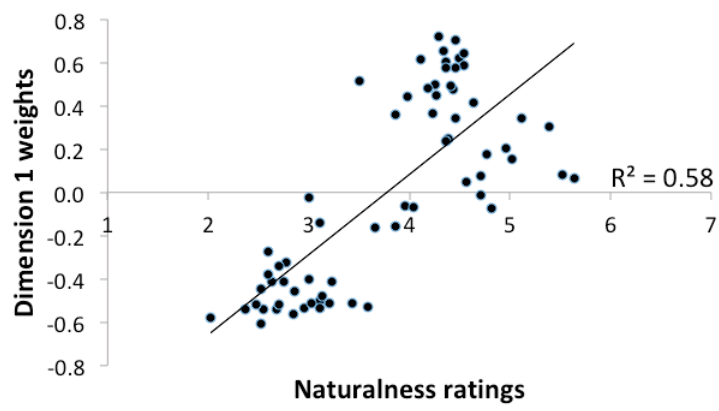

Dimension 1 weights vs. Naturalness ratings (Exteriors A)

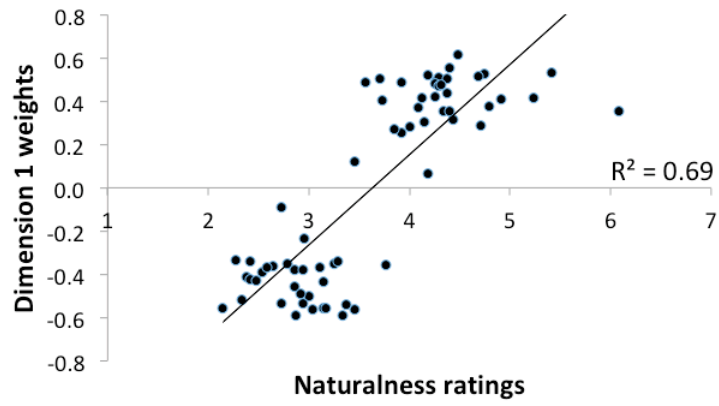

Dimension 1 weights vs. Naturalness ratings (Interiors B)

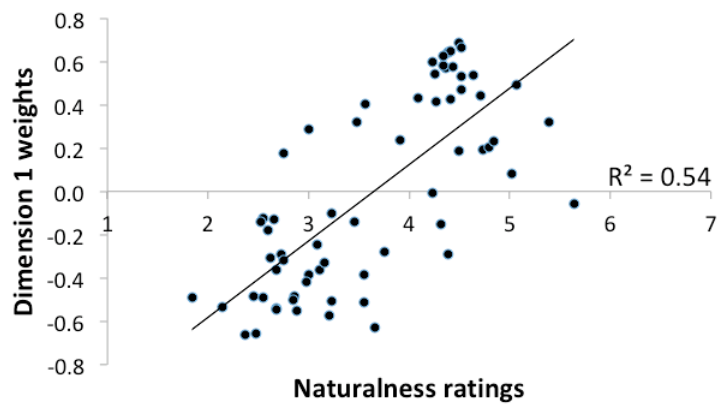

Dimension 1 weights vs. Naturalness ratings (Exteriors B)

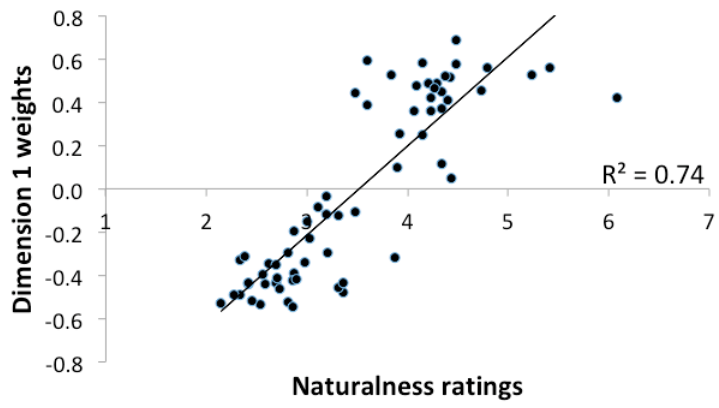

Figure 8: Correlations of Dimension 1 weights with Perceived Naturalness for the four sets of images.

The significant correlations between subjective naturalness ratings and Dimension 1 weights across all four sets of images suggest that MDS Dimension 1 is likely coding for the perceived naturalness of architectural scenes. In other words, Dimension 1 can be interpreted as representing a latent dimension of naturalness that influenced participants' judgments and image arrangement decisions as they were rating the similarity of architectural scenes. As such, these results show that participants "see" naturalness in these architectural scenes even though they were not primed to do so in any way.

\section{Scaling and Contrast patterns predict similarity ratings}

The preceding analyses have established that people have consistent perceptions of what they consider to be natural-looking architectural scenes, and that this aesthetic quality explains a substantial proportion of variance in similarity ratings of interior and exterior architectural scenes. Another important question we asked is whether similarity ratings (Dimension 1 weights) are driven by low-level visual patterns of Scaling and Contrast, which were found in the previous experiment to significantly predict perceptions of naturalness. We therefore conducted an analysis to see if these naturalistic patterns influenced perceptions of scene similarity. For this analysis, we regressed Dimension 1 weights on our Scaling feature 
(calculated in Experiment 1) and our three Contrast-related features (sdHue, sdSat, and sdBright). Explicit Nature was also added to the regression models to control for the amount of vegetation in the architectural scenes. Results of these regressions are shown in Tables 36.

Table 3: Regression of Dimension 1 weights vs. Scaling and Contrast-related features (Interiors A)

\begin{tabular}{lllllll}
\hline Visual Feature & Estimate & SE & $\boldsymbol{\beta}_{\text {ST }}$ & $\boldsymbol{t}$ value & $\boldsymbol{P}$ value & $\boldsymbol{n}_{\boldsymbol{p}}{ }^{2}$ \\
\hline Scaling*** & 0.296 & 0.035 & 0.689 & 8.511 & $<.001$ & 0.547 \\
sdHue & -0.038 & 0.031 & -0.086 & -1.225 & 0.225 & 0.024 \\
sdSat** & 0.090 & 0.033 & 0.211 & 2.727 & 0.008 & 0.110 \\
sdBright & 0.027 & 0.029 & 0.071 & 0.916 & 0.363 & 0.014 \\
Explicit Nature & -0.948 & 1.590 & -0.043 & -0.596 & 0.553 & 0.006 \\
\hline
\end{tabular}

$R^{2}{ }_{a d j}=0.68, F(5,60)=28.77, P<.001$

Table 4: Regression of Dimension 1 weights vs. Scaling and Contrast-related features (Interiors B)

\begin{tabular}{lllllll}
\hline Visual Feature & Estimate & SE & $\boldsymbol{\beta}_{\text {ST }}$ & $\boldsymbol{t}$ value & $\boldsymbol{P}$ value & $\boldsymbol{n}_{\boldsymbol{p}}{ }^{2}$ \\
\hline Scaling*** & 0.247 & 0.038 & 0.594 & 6.468 & $<.001$ & 0.411 \\
sdHue & -0.005 & 0.041 & -0.011 & -0.121 & 0.904 & 0.000 \\
sdSat* & 0.099 & 0.042 & 0.22 & 2.367 & 0.021 & 0.085 \\
sdBright & 0.051 & 0.035 & 0.134 & 1.463 & 0.149 & 0.034 \\
Explicit Nature & -3.359 & 2.082 & -0.142 & -1.613 & 0.112 & 0.042 \\
\hline
\end{tabular}

$R_{\text {adj }}^{2}=0.52, F(5,60)=15.02, P<.001$

Table 5: Regression of Dimension 1 weights vs. Scaling and Contrast-related features (Exteriors A)

\begin{tabular}{lclllll}
\hline Visual Feature & Estimate & SE & $\boldsymbol{\beta}_{\text {ST }}$ & t value & $\boldsymbol{P}$ value & $\boldsymbol{n}_{\boldsymbol{p}}{ }^{2}$ \\
\hline Scaling*** & 0.186 & 0.041 & 0.41 & 4.548 & $<.001$ & 0.256 \\
sdHue & 0.040 & 0.040 & 0.092 & 1.007 & 0.318 & 0.017 \\
sdSat** & 0.125 & 0.040 & 0.279 & 3.110 & 0.003 & 0.139 \\
sdBright*** & 0.179 & 0.042 & 0.413 & 4.302 & $<.001$ & 0.236 \\
Explicit Nature & -0.152 & 0.374 & -0.036 & -0.405 & 0.687 & 0.003 \\
\hline$R^{2}{ }_{\text {adj }}=0.57, F(5,60)=18.41, P<.001$ & & & & &
\end{tabular}

Table 6: Regression of Dimension 1 weights vs. Scaling and Contrast-related features (Exteriors B)

\begin{tabular}{lllllll}
\hline Visual Feature & Estimate & SE & $\boldsymbol{\beta}_{\text {ST }}$ & $\boldsymbol{t}$ value & $\boldsymbol{P}$ value & $\boldsymbol{n}_{\boldsymbol{p}}{ }^{2}$ \\
\hline Scaling* & 0.075 & 0.035 & 0.172 & 2.142 & 0.036 & 0.071 \\
sdHue* & 0.070 & 0.032 & 0.162 & 2.183 & 0.033 & 0.074 \\
sdSat*** & 0.193 & 0.035 & 0.519 & 5.529 & $<.001$ & 0.338 \\
sdBright* & 0.098 & 0.039 & 0.236 & 2.534 & 0.014 & 0.097 \\
Explicit Nature & 0.823 & 0.371 & 0.174 & 2.217 & 0.030 & 0.076 \\
\hline
\end{tabular}

$R^{2}{ }_{a d j}=0.67, F(5,60)=27.56, P<.001$

Scaling and Contrast-related features collectively explained over half of the variance in Dimension 1 weights in each image set. Two of these features, Scaling and sdSat, significantly predicted Dimension 1 weights across all four image sets. Furthermore, sdBright covaried significantly with Dimension 1 weights for both sets of exterior images, but not for interior 
scenes, and sdHue was a significant predictor of Dimension 1 weights for Exteriors B. These results suggest that Scaling and Contrast-related patterns may play an important role in driving similarity ratings of architectural scenes. These naturalistic visual features strongly predicted image similarity scores independent of how much vegetation was present in the architectural scenes, indicating that visual qualities of the buildings themselves influenced perceptions of scene similarity. In other words, people may unconsciously "see" these architectural patterns when arranging images of buildings without having been primed to do so.

\section{Experiment 3: \\ Does naturalness of buildings influence preference?}

Having established that participants "see" naturalness spontaneously (even while not necessarily aware of it) in purely architectural spaces, we set out to determine whether aesthetic preferences in the built environment are influenced by nature-like features of architectural design. We predicted that people would exhibit preferences for buildings that were perceived to be natural over artificial-looking spaces, and that nature-like patterns of Scaling and Contrast would correlate positively with aesthetic preference ratings. We also hypothesized that latent perceptions of naturalness would mediate the effect of Scaling and Contrast on aesthetic preference.

\section{Methods}

\section{Participants}

100 American adults (63 Women, 37 men) were recruited for this experiment from Amazon's Mechanical Turk (MTurk) to make preference ratings of the two architectural image sets. Sample size was determined by the goal of obtaining approximately 50 preference ratings per image (Kotabe et al., 2016b, 2017). Half of participants (Group 1) were assigned to rate images of interior spaces ( $n=50$ ), and the other half (Group 2 ) were assigned to rate images of exterior spaces $(n=50)$. Ages ranged from 20 to 60 years $(M=33.3, S D=9.5)$. Data was excluded from 4 participants who gave the same preference rating for 10 or more consecutive stimuli at least once during their individual trial, as this response pattern indicated that they were not attending to the task. All participants were compensated $\$ 1.00$ for their participation and the experiment took approximately 10 minutes to complete. Informed consent was obtained through the Institutional Review Board (IRB) of the University of Chicago.

\section{Procedure}

Participants rated the interior and exterior image sets from Experiment 1 using the online interface of Qualtrics survey software. Group 1 participants $(n=50)$ were asked to rate how 
much they liked each interior image using a Likert scale ranging from 1 to 7 , with 1 indicating strong dislike and 7 indicating strong preference. Group 2 participants $(n=50)$ followed the same procedure but made preference ratings for images of architectural exteriors rather than interiors.

\section{Statistical analysis}

We conducted analyses at the image level by calculating average preference ratings for each image across all participants, and by using image-level naturalness scores obtained in Experiment 1. First, we regressed preference ratings on naturalness ratings using a simple linear regression model. Next, we conducted mediation analyses to examine whether latent perceptions of naturalness mediated the influence of Scaling and Contrast-related scene feature on aesthetic preferences. In order to simplify the mediation analysis for Contrast, a Principal Components Analysis was carried out on the three Contrast-related features (sdSat, sdHue, and sdBright) and the first principle component was used as a single-variable proxy for visual contrast, which we labeled Contrast. We quantified latent perceptions of naturalness by calculating the variance in Dimension 1 weights (from Experiment 2) predicted by naturalness ratings. This new variable was labeled Implicit Naturalness. Mediation analyses were then conducted to investigate whether Implicit Naturalness mediated the effect of Scaling and Contrast on aesthetic preference ratings for each image set.

\section{Results}

\section{Naturalness predicts Preference}

The first analysis explored the degree to which the mean naturalness ratings of interior and exterior images predicted mean preference ratings. The perception of naturalness strongly predicted preference for both architectural interiors $\left[R^{2}=0.70, F(1,118)=275, P<.001, \beta_{\mathrm{ST}}\right.$ $=0.836, t=16.58]$ and exteriors $\left[R^{2}=0.45, F(1,118)=96.1, P<.001, \beta_{\mathrm{ST}}=0.670, t=9.81\right]$. These results suggest that architectural scenes with more naturalistic qualities are preferred, on average, over scenes that are perceived as more artificial. This finding extends past empirical work linking naturalness and preference (S. Kaplan et al., 1972; Kardan, Demiralp, et al., 2015) to the context of architectural scenes.

\section{Naturalness mediates the effect of Scaling on Preference}

Next, we investigated whether Scaling patterns influenced aesthetic preference ratings, and we tested whether this effect was mediated by latent perceptions of naturalness. We operationalized latent perceptions of naturalness by calculating the MDS Dimension 1 
weights (from Experiment 2) predicted by naturalness ratings (from Experiment 1). ${ }^{12} \mathrm{We}$ labeled this new variable Implicit Naturalness because it captures the variance in implicit evaluations of image similarity explained by naturalness ratings. A diagram of the hypothesized mediation model is illustrated in Figure 9. Linear regressions revealed that Scaling significantly predicted preference for both architectural interiors $[B=0.666, \mathrm{SE}=$ $0.066, \mathrm{t}=10.13 P<.001]$ and exteriors $[B=0.539, \mathrm{SE}=0.086, \mathrm{t}=6.26, P<.001]$. However, the direct effect of Scaling on Preference was significantly reduced when controlling for the mediator, Implicit Naturalness, for both interiors $[B=0.211$, SE $=0.063, \mathrm{t}=3.38, P=0.001]$ and exteriors $[B=0.230, \mathrm{SE}=0.083, \mathrm{t}=2.78, P<0.01]$. We tested the indirect effect of the mediation pathway using a bootstrap estimation approach with 10,000 samples (Shrout \& Bolger, 2002). These results indicated that the indirect coefficient was significant for interiors $[B=0.455, \mathrm{SE}=0.052,95 \% \mathrm{Cl}=0.360,0.565]$ and exteriors $[B=0.309, \mathrm{SE}=0.050,95 \% \mathrm{Cl}=$ $0.222,0.418]$. These results suggest that Implicit Naturalness partially mediates the effect of Scaling on Preference for both image sets. In other words, architectural scenes with greater Scaling are preferred, in part, because they are perceived as more natural-looking than those with less Scaling. The results of these analyses are shown in Figure 10.

Figure 9: Mediation models. (A) Diagram of Total Effect (no mediators). (B) Diagram of mediation model with Naturalness as mediator. Note the Direct Effect and the Indirect Effect (i.e. mediation pathway).

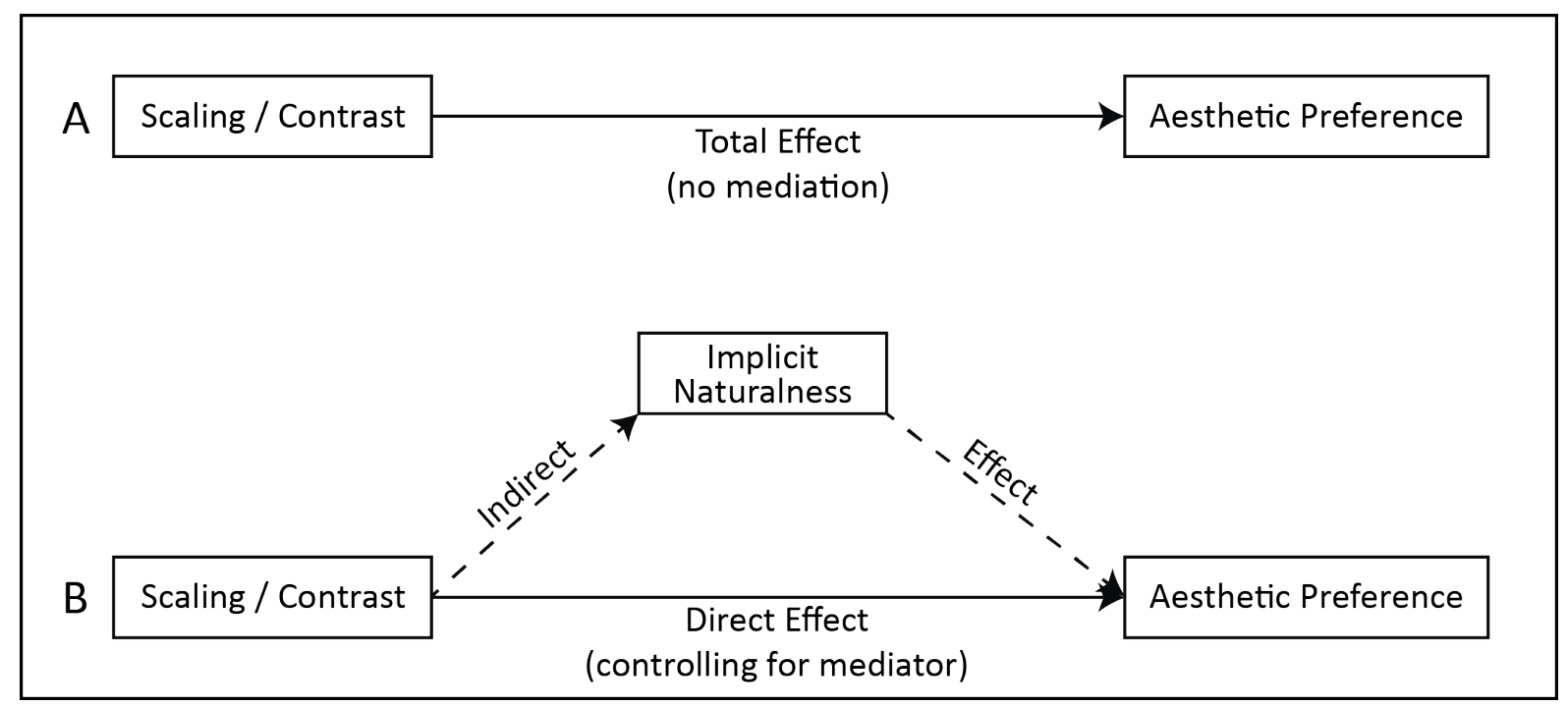

Naturalness mediates the effect of Contrast on Preference

\footnotetext{
${ }^{12}$ We did this by constructing a linear regression model of MDS Dimension 1 weights $(\mathrm{Y})$ vs. Naturalness ratings $(\mathrm{X})$. Then, we subtracted the residuals of this regression model from Dimension 1 weights, thus yielding the Dimension 1 weights predicted by Naturalness ratings.
} 
Finally, we investigated whether Implicit Naturalness mediated the effect of Contrast on Preference. Instead of carrying out three separate mediations for each image set, we reduced the three Contrast-related variables (sdHue, sdSat, sdBright) to one variable using Principal Components Analysis (PCA). A PCA was carried on these three variables for both image sets. The first principal component (PC1), which explained $47 \%$ of variance for interiors and $54 \%$ of variance for exteriors, was used as a proxy for Contrast. Linear regressions indicated that Contrast (PC1) significantly predicted Preference in both image sets [Interiors: $B=0.488, \mathrm{SE}=$ $0.082, \mathrm{t}=5.98, P<.001$; Exteriors: $B=0.614, \mathrm{SE}=0.076, \mathrm{t}=8.09]$. For interior scenes, Contrast was no longer a significant predictor of Preference when controlling for the mediator, Implicit Naturalness $[B=-0.044, \mathrm{SE}=0.064, \mathrm{t}=-0.69, P=0.49]$. For exteriors scenes, the direct effect of Contrast on Preference was significantly reduced when controlling for Implicit Naturalness $[B=0.281, \mathrm{SE}=0.088, \mathrm{t}=3.19, P=0.002]$. The indirect effect of the mediation pathway was tested using a bootstrap estimation approach with 10,000 samples (Shrout \& Bolger, 2002). The indirect coefficient was significant for both image sets [Interiors: $B=0.531, \mathrm{SE}=0.071$, $95 \% \mathrm{Cl}=0.402,0.679$; Exteriors: $B=0.333, \mathrm{SE}=0.066,95 \% \mathrm{Cl}=0.219,0.477]$. These results suggest that the effect of Contrast on Preference is fully mediated by latent perceptions of naturalness for interiors and partially mediated by latent perceptions of naturalness for exteriors. Results of these analyses are displayed in Figure 10.

Figure 10: Results of mediation analyses.

\begin{tabular}{|c|c|c|c|c|}
\hline & Feature & $\begin{array}{l}\text { Total Effect } \\
(95 \% \mathrm{Cl})\end{array}$ & $\begin{array}{l}\text { Direct Effect } \\
\qquad(95 \% \mathrm{Cl})\end{array}$ & $\begin{array}{c}\text { Indirect Effect } \\
(95 \% \mathrm{Cl})\end{array}$ \\
\hline \multirow{2}{*}{ 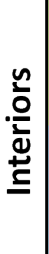 } & Scaling & $\begin{array}{c}\mathbf{0 . 6 6 6} \\
(0.536,0.797)\end{array}$ & $\begin{array}{c}\mathbf{0 . 2 1 1} \\
(0.087,0.335)\end{array}$ & $\begin{array}{c}\mathbf{0 . 4 5 5} \\
(0.360,0.565)\end{array}$ \\
\hline & Contrast & $\begin{array}{c}\mathbf{0 . 4 8 8} \\
(0.326,0.649)\end{array}$ & $\begin{array}{c}-\mathbf{0 . 0 4 4} \\
(-0.170,0.083)\end{array}$ & $\begin{array}{c}\mathbf{0 . 5 3 1} \\
(0.402,0.679)\end{array}$ \\
\hline \multirow{3}{*}{ 号 } & Scaling & $\begin{array}{c}\mathbf{0 . 5 3 9} \\
(0.369,0.710)\end{array}$ & $\begin{array}{c}\mathbf{0 . 2 3 0} \\
(0.066,0.394)\end{array}$ & $\begin{array}{c}\mathbf{0 . 3 0 9} \\
(0.222,0.418)\end{array}$ \\
\hline & Contrast & $\begin{array}{c}\mathbf{0 . 6 1 4} \\
(0.464,0.764)\end{array}$ & $\begin{array}{c}\mathbf{0 . 2 8 1} \\
(0.107,0.455)\end{array}$ & $\begin{array}{c}\mathbf{0 . 3 3 3} \\
(0.219,0.477)\end{array}$ \\
\hline & & Partial Mediation & Full Mediation & \\
\hline
\end{tabular}

\section{Discussion}

In this study, we investigated whether subjective measures of naturalness in the built environment are driven by objective low-level features of architectural images, and we examined the degree to which nature-like architectural features influence similarity evaluations and preference ratings for interior and exterior architectural scenes. Our first 
experiment revealed that perceptions of naturalness covaried significantly with low-level spatial and color features of images and were significantly associated with two of Alexander's proposed patterns of natural structure, Levels of Scale and Contrast (Alexander, 2002), across both image sets. Image similarity scores derived from an image arrangement task in our second experiment correlated highly with subjective naturalness ratings, suggesting that people may intuitively organize and evaluate architectural images based on latent perceptions of naturalness. In other words, people utilize naturalness as a grouping method in architectural scenes spontaneously and automatically even when they are not primed to do so in any way. Results from our final experiment indicated that Scaling and Contrast patterns strongly predicted aesthetic preference ratings across both image sets, and that the effects of these patterns on preferences were mediated by latent perceptions of naturalness in architectural scenes.

Results from Experiment 1 build on previous findings showing that subjective perceptions of naturalness are influenced by low-level spatial and color features for outdoor scenes (Berman et al., 2014; Kardan, Demiralp, et al., 2015), many of which had little to no built structure. Here, we extend these findings to architectural scenes, many of which had little to no vegetated content, demonstrating that perceptions of whether a building looks natural or artificial can also be reliably predicted by low-level scene features. Image statistics in our regression models independently predicted $54 \%$ of variance in naturalness ratings for interior scenes and $42 \%$ of variance for exterior scenes when controlling for the amount of vegetation visible in the scenes. Interestingly, the same three visual features (Scaling, sdSat, and sdBright) significantly predicted naturalness scores for both interior and exterior architectural stimuli. Furthermore, two of these features (Scaling ${ }^{13}$ and sdSat) were previously shown to drive perceptions of naturalness in outdoor scenes (Berman et al., 2014; Kardan, Demiralp, et al., 2015). The consistency of these results suggests that certain sensory qualities associated with naturalness may transcend scene categories. In other words, visual patterns that make landscapes feel natural can potentially be reproduced in architecture, making some buildings feel as if they emerged organically from the earth.

This idea echoes the general philosophy outlined in the Nature of Order books (Alexander, 2002), which argues that buildings develop nature-like visual patterns when the processes by which they are constructed resemble biological growth. Although evaluating construction processes was beyond the scope of our study, Experiment 1 enabled us to measure two of these proposed nature-like patterns, Levels of Scale and Contrast, and test whether they

\footnotetext{
13 In the previous study, Scaling was not directly measured. Rather, measures of Edge Density correlated positively with naturalness. However, these measures are nearly identical, given that our Scaling measure was a composite of Edge Density and Fractal Dimension, which were highly correlated across both of our image sets.
} 
determined subjective naturalness ratings of architectural scenes. Our Scaling measure and two of our three contrast measures (sdSat and sdBright) significantly predicted naturalness ratings for both interior and exterior image sets, supporting the hypothesis that buildings exhibiting more Levels of Scale and greater visual Contrast tend to look more natural.

These patterns predicted naturalness ratings even when controlling for Explicit Nature, i.e., the presence of vegetation, indicating that naturalistic visual patterns inherent in the buildings themselves were likely driving perceptions of naturalness. This finding supports the idea that perceptions of naturalness may not depend exclusively on conscious recognition of nature-related semantic content in an environment, but may also be driven by intuitive responses to architectural patterns that evoke unconscious associations with the natural world (Alexander, 2002; Kellert, 2005).

Experiment 2 enabled us to test the saliency of the aesthetic quality of naturalness in architectural scenes by investigating whether scene similarity judgements obtained from an image-arrangement task were driven by latent perceptions of naturalness. Subjective naturalness ratings collected in the previous experiment significantly predicted over half of the variance in MDS Dimension 1 weights for both interior image sets and over two-thirds of variance in MDS Dimension 1 weights for both exterior image sets. Based on these data, participants seemed to intuitively evaluate architectural scenes according to their degree of naturalness while making similarity judgements, even though they were not prompted to evaluate the naturalness of the scenes in any way. These results build on previous work showing that people unconsciously rely on perceptions of naturalness to evaluate the similarity of outdoor scenes containing relatively little built structure (Berman et al., 2014). Here, we extend those findings to the context of architectural spaces, showing that naturalness is also an important perceptual dimension of architectural scenes containing relatively little vegetation.

Further regression analyses in Experiment 2 showed that, in all four image sets, Scaling and Contrast-related visual features explained more than half of the variance in Dimension 1 weights when controlling for the amount of vegetation depicted in scenes (Explicit Nature). These results suggest that people may be particularly attuned to these low-level architectural patterns when evaluating scene similarity. It is important to note, however, that a large portion of variance in Dimension 1 weights was not captured by any of the independent variables included in the analysis, suggesting that people likely relied on other aesthetic scene features, in addition to natural patterns, when judging image similarity.

After establishing that people spontaneously use naturalness to organize purely architectural scenes, we carried out a third experiment to test whether people exhibit innate preferences for scenes exhibiting nature-like architectural patterns of Scaling and Contrast. These visual patterns significantly predicted preference ratings in both image sets. Furthermore, mediation analyses demonstrated that the effects of Scaling and Contrast features on 
preference ratings were either partially or fully mediated by latent perceptions of naturalness, as operationalized by our Implicit Naturalness variable, for both interior and exterior scenes. These results suggest that architectural patterns of Scaling and Contrast may generate aesthetic pleasure because they are reminiscent of visual patterns often found in nature.

The idea that humans are innately drawn to nature-inspired architectural forms dates back several centuries. Immanuel Kant believed that man's most beautiful creations look as if they emerged organically from the earth, because they reflect the artist's intuitive understanding of nature's underlying order. Philosopher John Ruskin later wrote that "whatever is in architecture fair or beautiful is imitated from natural forms" (Ruskin, 1849, p. 71). Extending these ideas to the scientific realm, contemporary proponents of biophilic design often contend that humans have developed innate affinities for naturalistic forms in their surroundings over the course of evolutionary history (Joye, 2007b; Salingaros, 2007; Wilson, 1984; Wilson \& Kellert, 1995), and that nature-inspired architectural features may foster important psychological benefits (Alexander, 2002; Joye, 2007a; Kellert, 2005; Salingaros, 1998). ${ }^{14}$ The experiments presented here offer among the first empirical evidence that naturalistic patterns in architecture may be inherently preferred over synthetic forms and suggest that the biophilia phenomenon may extend into the built environment. By quantifying two low-level patterns characteristic of naturalistic architecture - Levels of Scale and Contrast - this study also paves the way for future researchers to investigate whether variations in these patterns might enhance mood, cognitive functioning, or other aspects of psychological experience.

It is especially relevant today to ask whether naturalness in the built environment confers benefits to aesthetic experience and psychological functioning, given that "modern habitats contain increasingly less actual nature or artistic references to natural form" (Joye, 2007b, p. 323). Indeed, many contemporary architectural movements have abandoned the "monumental design model" (Kellert, 2003, p. 36) of nature that previously inspired traditional building styles across the globe. Some scholars have speculated that the absence of biophilic features in many of today's homes, offices, and urban neighborhoods may foster detrimental psychological and social experiences (Alexander, 2002; Joye, 2007b; Kellert, 2005; Salingaros, 2007). Kellert, for example, writes that "the quality and character of the contemporary built environment has increasingly isolated people from the beneficial experience of natural systems and processes" (Kellert, 2005, p. 123) and asserts that inorganic

\footnotetext{
${ }^{14}$ Kellert, for instance, writes that "organic qualities of light, color, material, texture, shape, and form that have been symbolically borrowed from the natural world... grip the human imagination, communicating a subtle connection to the natural environment, even when the origin of our feelings remains obscure" (Kellert, 2005, p. 159)
} 
design contributes to "the alienation associated with much contemporary architecture" (Kellert, 2005, p. 133). These claims, however, have yet to be empirically tested.

This study provides useful tools for researchers interested in exploring these ideas empirically and for architects wishing to incorporate biophilic design features into the built environment. The two nature-like patterns that we tested in our study - Levels of Scale and Contrast - can be manipulated in architectural design schemes. They are also non-prescriptive, as they do not require adherence to a particular architectural style. The results of these experiments also highlight the importance of investigating how Alexander's other proposed patterns of natural structure (Alexander, 2002) and adaptive construction processes (Alexander, 2004) might impact various aspects of psychological experience, compared to more conventional approaches to architectural design and construction. These questions could be addressed in future studies that evaluate psychological and behavioral responses to varying design patterns in real or virtual environments.

\section{Limitations}

We relied on images of buildings as our stimuli for these studies in order to isolate scene features related to visual perception and to expose participants to a wide variety of architectural spaces within a reasonable timeframe. However, using two-dimensional stimuli may limit the generalizability of these findings to three-dimensional built spaces. We also focused here on visual perception of architecture. In doing so, we were unable to make inferences about the contribution of nonvisual sensory features of architectural spaces to perceptions of naturalness, scene similarity, and preference, which we would expect to be substantial. Finally, our dependent variables did not directly test the psychological benefits of naturalistic patterns in buildings. Future studies could use tests of cognitive function, such as working memory tasks, to address more directly whether biophilic features of architecture offer measurable benefits to mental health.

\section{Conclusion}

These experiments attempt to operationalize the aesthetic quality of naturalness in the built environment. We offer among the first empirical evidence that natural patterns inherent in low-level visual features, such as Scaling and Contrast, may influence similarity evaluations and preference ratings of architectural scenes. This evidence builds on a wealth of theoretical work suggesting that humans may be innately attuned to biophilic sensory features of the built environment. By identifying specific spatial and color patterns that are perceived as more natural, and which can easily be manipulated in architectural design, this research empowers architects with flexible tools for designing biophilic spaces and enables researchers to test whether nature-like architectural features might contribute to restorative psychological experiences. 


\section{Acknowledgements}

Special thanks to Tanvi Lakhtakia, Anabella Pinton, Claire Sampson, Leah Stiff, Taj Ebanks, and Kathryn Schertz, all of whom made a valuable contribution to this paper by collecting data for Experiment 2. This research was supported by generous funding from the TKF Foundation, the John Templeton Foundation (University of Chicago Center for Practical Wisdom and the Virtue, Happiness, and Meaning of Life Scholars Group), NSF Grant BCS-1632445 to MGB, the University of Chicago College Research Fellows Program, and the Cambridge Trusts. The funders had no role in study design, data collection, data analysis, manuscript preparation, or publication. 


\section{Supplemental Materials}

Table S1: Deviation Contrasts for ANOVA of Naturalness vs. Building Type (interiors)

\begin{tabular}{lllll}
\hline Comparison & Estimate & SE & $\boldsymbol{t}$ value & $\boldsymbol{p}$ value \\
\hline Educational - Mean & -0.022 & 0.18 & -0.12 & 0.904 \\
Government - Mean & 0.174 & 0.18 & 0.968 & 0.335 \\
Medical - Mean & 0.32 & 0.18 & 1.785 & 0.077 \\
Religious - Mean & -0.206 & 0.18 & -1.146 & 0.254 \\
Residential - Mean & -0.194 & 0.18 & -1.082 & 0.281 \\
\hline
\end{tabular}

$\eta_{p}^{2}=0.047, F(5,114)=1.31, p=0.35$

Table S2: Deviation Contrasts for ANOVA of Preference vs. Building Type (interiors)

\begin{tabular}{lllll}
\hline Comparison & Estimate & SE & $\boldsymbol{t}$ value & $\boldsymbol{p}$ value \\
\hline Educational - Mean & 0.371 & 0.213 & 1.745 & 0.084 \\
Government - Mean & 0.138 & 0.213 & 0.647 & 0.519 \\
Medical - Mean & 0.048 & 0.213 & 0.225 & 0.822 \\
Religious - Mean & -0.29 & 0.213 & -1.361 & 0.176 \\
Residential - Mean & -0.278 & 0.213 & -1.304 & 0.195 \\
\hline
\end{tabular}

$\eta_{p}^{2}=0.049, F(5,114)=1.18, p=0.32$

Table S3: Deviation Contrasts for ANOVA of Naturalness vs. Building Type (exteriors)

\begin{tabular}{lllll}
\hline Comparison & Estimate & SE & $\boldsymbol{t}$ value & $\boldsymbol{p}$ value \\
\hline Educational - Mean & -0.173 & 0.165 & -1.051 & 0.296 \\
Government - Mean & 0.104 & 0.165 & 0.63 & 0.53 \\
Medical - Mean & 0.193 & 0.165 & 1.173 & 0.243 \\
Religious - Mean & -0.191 & 0.165 & -1.158 & 0.249 \\
Residential - Mean & 0.002 & 0.165 & 0.011 & 0.992 \\
\hline
\end{tabular}

$\eta^{2}=0.031, F(5,114)=0.73, p=0.60$

Table S4: Deviation Contrasts for ANOVA of Preference vs. Building Type (exteriors)

\begin{tabular}{lllll}
\hline Comparison & Estimate & SE & t value & $\boldsymbol{p}$ value \\
\hline Educational - Mean & -0.094 & 0.219 & -0.43 & 0.668 \\
Government - Mean & -0.29 & 0.219 & -1.322 & 0.189 \\
Medical - Mean & 0.167 & 0.219 & 0.763 & 0.447 \\
Religious - Mean & -0.351 & 0.219 & -1.598 & 0.113 \\
Residential - Mean & 0.43 & 0.219 & 1.96 & 0.052 \\
\hline
\end{tabular}

$\eta^{2}=0.064, F(5,114)=1.55, p=0.18$ 


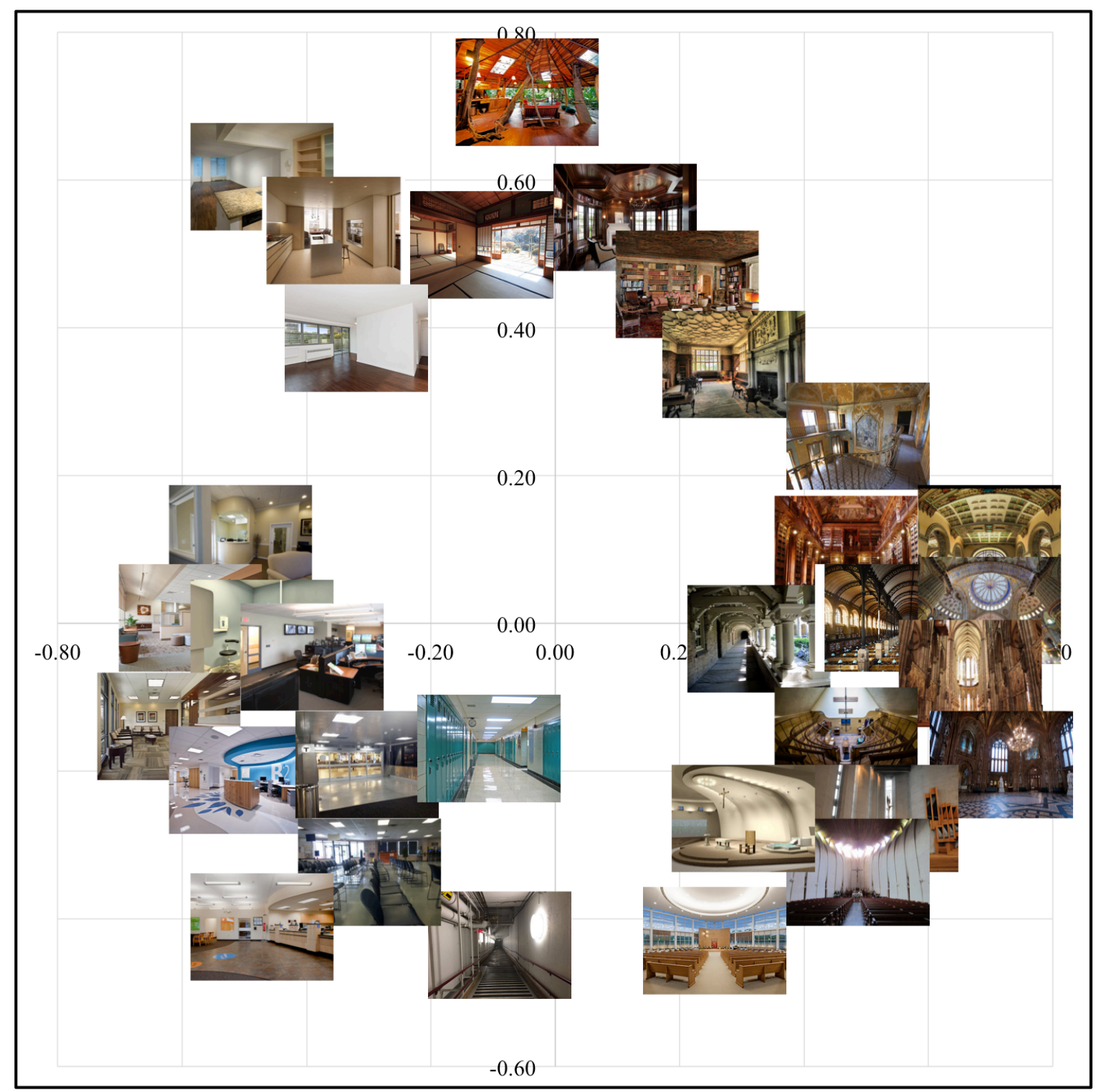

Figure 11: Plotted results of MDS dimension 1 (X-axis) and Dimension 2 (Y-axis) for the second image set (Interiors B). Pictures are superimposed based on their weights on Dimensions 1 and 2. A subset of the 66 images is plotted in order to make the graph more readable. 


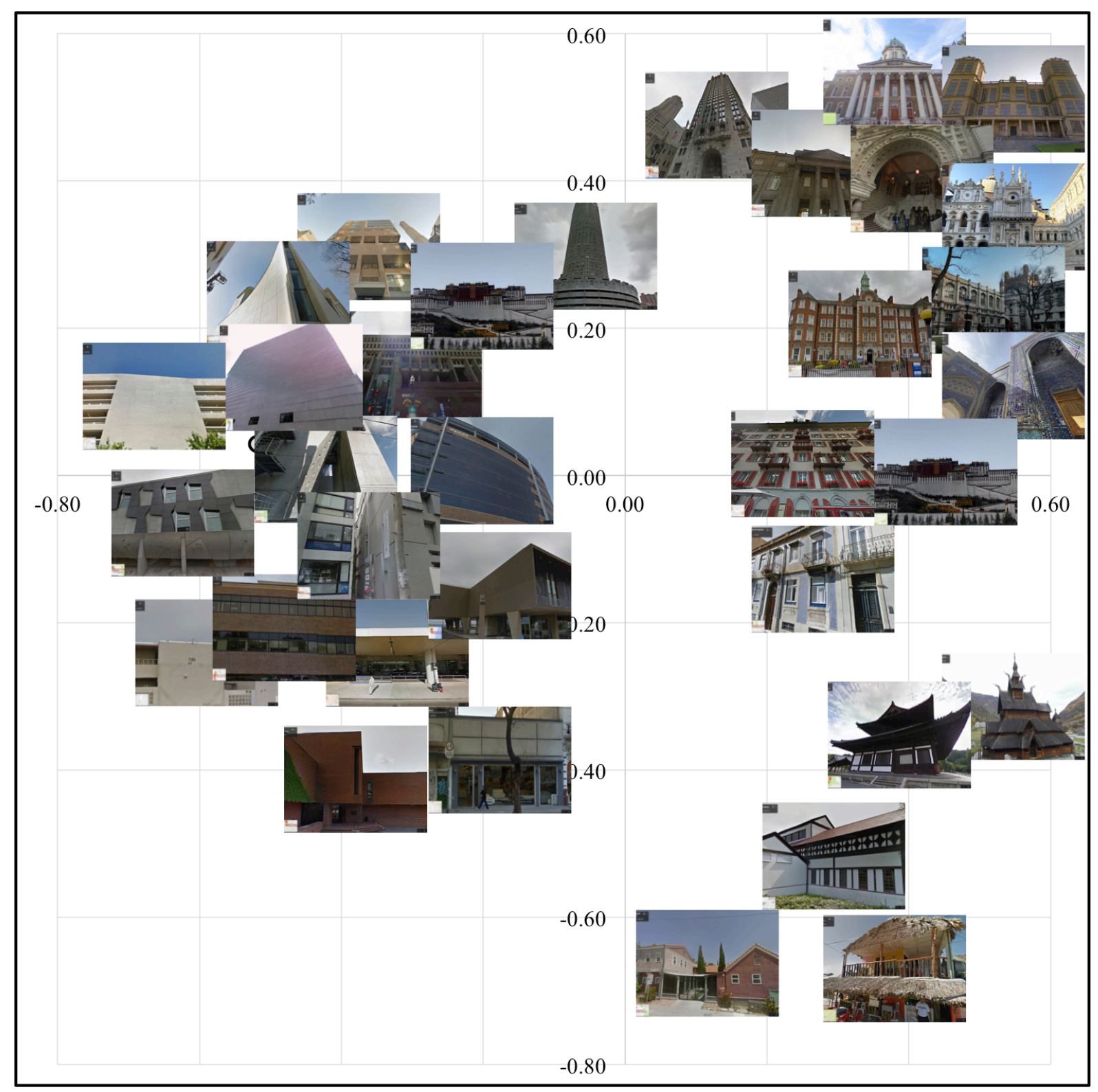

Figure 12: Plotted results of MDS dimension 1 (X-axis) and Dimension 2 (Y-axis) for the thrid image set (Exteriors A). Pictures are superimposed based on their weights on Dimensions 1 and 2. A subset of the 66 images is plotted in order to make the graph more readable. 


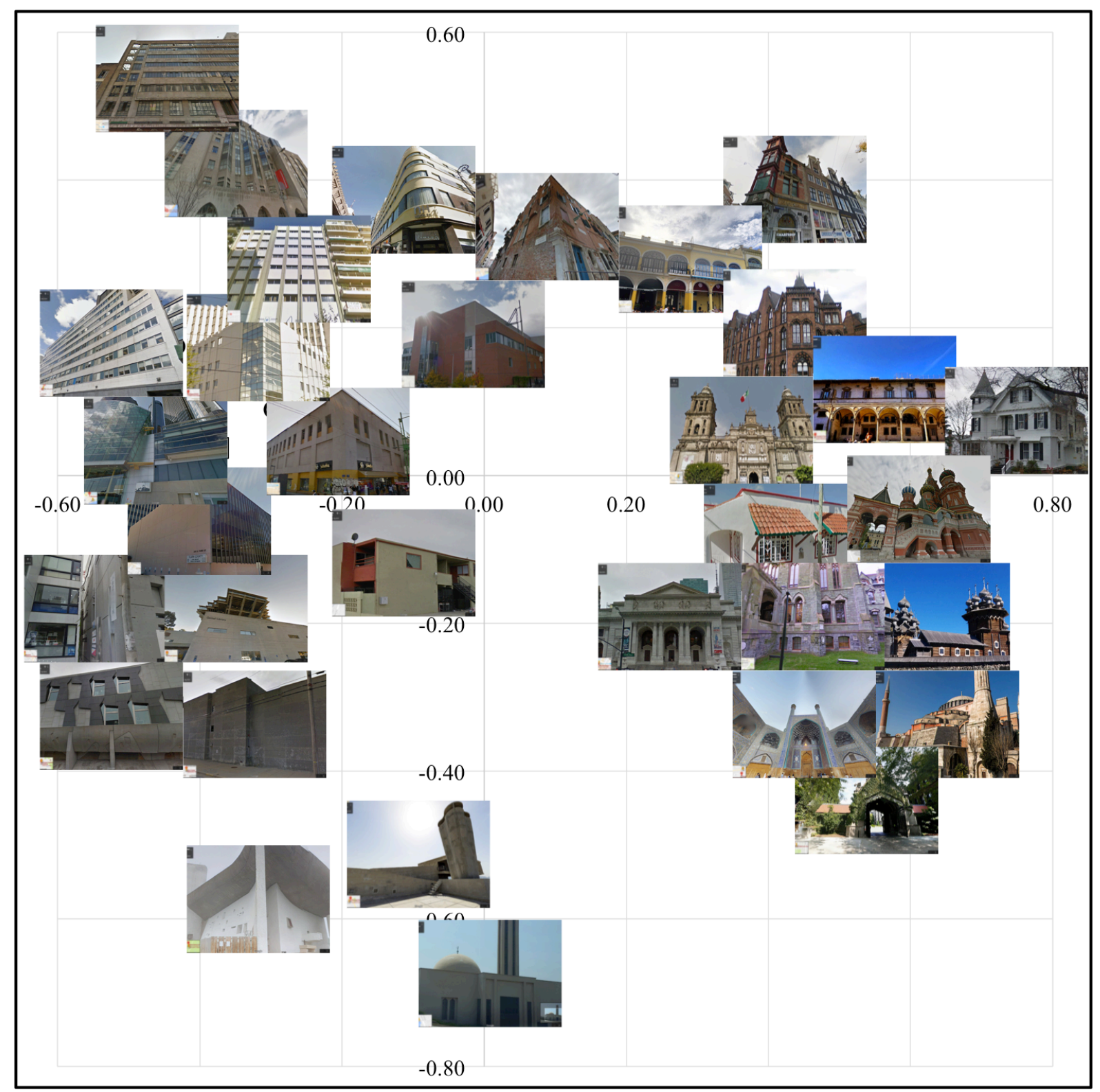

Figure 13: Plotted results of MDS dimension 1 (X-axis) and Dimension 2 (Y-axis) for the fourth image set (Exteriors B). Pictures are superimposed based on their weights on Dimensions 1 and 2. A subset of the 66 images is plotted in order to make the graph more readable. 


\section{References}

Adams, M. (2014). Quality of urban spaces and wellbeing. In Wellbeing and the Environment (Vol. 2, pp. 249-270). West Sussex, England: John Wiley \& Sons Inc.

Aldersey-Williams, H. (2004, May 1). Towards biomimetic architecture [Comments and Opinion]. doi:10.1038/nmat1119

Alexander, C. (2002). The phenomenon of life: an essay on the art of building and the nature of the universe (Vol. 1). Berkeley, Calif: Center for Environmental Structure.

Alexander, C. (2004). The Process of Creating Life: The Nature of Order, Book 2: An Essay of the Art of Building and the Nature of the Universe: Bk. 2. Berkeley, Calif: Routledge.

Barton, J., \& Pretty, J. (2010). What is the best dose of nature and green exercise for improving mental health? A multi-study analysis. Environmental Science \& Technology, 44, 3947.

Berman, M. G., Hout, M. C., Kardan, O., Hunter, M. R., Yourganov, G., Henderson, J. M., ... Jonides, J. (2014). The Perception of Naturalness Correlates with Low-Level Visual Features of Environmental Scenes. PLOS ONE, 9, e114572.

Berman, M. G., Jonides, J., \& Kaplan, S. (2008). The Cognitive Benefits of Interacting with Nature. Psychological Science, 19, 1207-1212.

Berman, M. G., Kross, E., Krpan, K. M., Askren, M. K., Burson, A., Deldin, P. J., ... Jonides, J. (2012). Interacting with nature improves cognition and affect for individuals with depression. Journal of Affective Disorders, 140, 300-305.

Berto, R. (2005). Exposure to restorative environments helps restore attentional capacity. Journal of Environmental Psychology, 25, 249-259.

Bowler, D. E., Buyung-Ali, L. M., Knight, T. M., \& Pullin, A. S. (2010). A systematic review of evidence for the added benefits to health of exposure to natural environments. BMC Public Health, 10, 1-10.

Bratman, G. N., Daily, G. C., Levy, B. J., \& Gross, J. J. (2015). The benefits of nature experience: Improved affect and cognition. Landscape and Urban Planning, 138, 41-50.

Bratman, G. N., Hamilton, J. P., \& Daily, G. C. (2012). The impacts of nature experience on human cognitive function and mental health. Annals of the New York Academy of Sciences, $1249,118-136$.

Bratman, G. N., Hamilton, J. P., Hahn, K. S., Daily, G. C., \& Gross, J. J. (2015). Nature experience reduces rumination and subgenual prefrontal cortex activation. Proceedings of the National Academy of Sciences, 112, 8567-8572. 
Busing, F., Commandeur, J. J., Heiser, W. J., Bandilla, W., \& Faulbaum, F. (1997). PROXSCAL: A multidimensional scaling program for individual differences scaling with constraints.

Sofstat, 97, 67-74.

Coburn, A., Vartanian, O., \& Chatterjee, A. (2017). Buildings, Beauty, and the Brain: A Neuroscience of Architectural Experience. Journal of Cognitive Neuroscience, 29, 15211531.

Cooper, R., \& Burton, E. (2014). Wellbeing and the environmental implications for design. In Wellbeing and the Environment (Vol. 2, pp. 653-668). West Sussex, England: John Wiley \& Sons Inc.

Evans, G. W., \& McCoy, J. M. (1998). When buildings don't work: The role of architecture in human health. Journal of Environmental Psychology, 18, 85-94.

Forget, Y. (2009). Taj Mahal [Photograph]. Retrieved from https://en.wikipedia.org/wiki/Taj_Mahal\#/media/File:Taj_Mahal_(Edited).jpeg

Goldberger, A. L. (1996). Fractals and the birth of Gothic: Reflections on the biologic basis of creativity. Molecular Psychiatry, 1, 99-104.

Hartig, T. (2008). Green space, psychological restoration, and health inequality. The Lancet, $372,1614-1615$.

Horsley, D. (2017). Generalising Fisher's inequality to coverings and packings.

Combinatorica, 37, 673-696.

Hout, M. C., Cunningham, C., Robbins, A., \& MacDonald, J. (2018). Simulating the fidelity of data for large stimulus set sizes and variable dimension estimation in multidimensional scaling. Sage Open.

Hout, M. C., Godwin, H. J., Fitzsimmons, G., Robbins, A., Menneer, T., \& Goldinger, S. D. (2015). Using multidimensional scaling to quantify similarity in visual search and beyond. Attention, Perception, \& Psychophysics, 78, 3-20.

Hout, M. C., \& Goldinger, S. D. (2016). SpAM is convenient, but also satisfying: Reply to Verheyen et al. (2016). Journal of Experimental Psychology: General, 3, 383-387.

Hout, M. C., Goldinger, S. D., \& Ferguson, R. W. (2013). The versatility of SpAM: A fast, efficient spatial method of data collection for multidimensional scaling. Journal of Experimental Psychology: General, 142, 256-281.

Hout, M. C., Papesh, M. H., \& Goldinger, S. D. (2013). Multidimensional scaling. Wiley Interdisciplinary Reviews: Cognitive Science, 4, 93-103. 
Ibarra, F. F., Kardan, O., Hunter, M. R., Kotabe, H. P., Meyer, F. A. C., \& Berman, M. G. (2017). Image Feature Types and Their Predictions of Aesthetic Preference and Naturalness. Frontiers in Psychology, 8. doi:10.3389/fpsyg.2017.00632

Joye, Y. (2006). An interdisciplinary argument for natural morphologies in architectural design. Environment and Planning B: Planning and Design, 33, 239-252.

Joye, Y. (2007a). A tentative argument for the inclusion of nature-based forms in architecture. Unpublished Doctoral Dissertation, Ghent University, Ghent, Belgium.

Retrieved from

http://citeseerx.ist.psu.edu/viewdoc/download?doi=10.1.1.468.7160\&rep=rep1\&type=pdf

Joye, Y. (2007b). Architectural lessons from environmental psychology: The case of biophilic architecture. Review of General Psychology, 11, 305-328.

Joye, Y., Steg, L., Ünal, A. B., \& Pals, R. (2016). When complex is easy on the mind: Internal repetition of visual information in complex objects is a source of perceptual fluency. Journal of Experimental Psychology: Human Perception and Performance, 42, 103.

Kant, I. (2001). Critique of the Power of Judgment. (P. Guyer \& E. Matthews, Trans.) (Revised edition). Cambridge: Cambridge University Press.

Kaplan, S. (1995). The restorative benefits of nature: Toward an integrative framework. Journal of Environmental Psychology, 15, 169-182.

Kaplan, S., \& Berman, M. G. (2010). Directed Attention as a Common Resource for Executive Functioning and Self-Regulation. Perspectives on Psychological Science, 5, 43-57.

Kardan, O., Demiralp, E., Hout, M. C., Hunter, M. R., Karimi, H., Hanayik, T., ... Berman, M. G. (2015). Is the preference of natural versus man-made scenes driven by bottom-up processing of the visual features of nature? Frontiers in Psychology, 6.

doi:10.3389/fpsyg.2015.00471

Kardan, O., Gozdyra, P., Misic, B., Moola, F., Palmer, L. J., Paus, T., \& Berman, M. G. (2015). Neighborhood greenspace and health in a large urban center. Scientific Reports, 5, 11610.

Karmanov, D., \& Hamel, R. (2008). Assessing the restorative potential of contemporary urban environment(s): Beyond the nature versus urban dichotomy. Landscape and Urban Planning, 86, 115-125.

Kellert, S. R. (2003). Kinship to mastery: Biophilia in human evolution and development. Island Press. Retrieved from https://books.google.com/books?hl=en\&lr=\&id=PkufpPphlz8C\&oi=fnd\&pg=PP15\&dq=kinshi $\mathrm{p}+$ to+master+biophilia+in+human+evolution\&ots=f6ZIC-

VdnC\&sig=3SfZaMmDbhuArghgLp9y9iqZ3Gc 
Kellert, S. R. (2005). Building for life: designing and understanding the human-nature connection. Washington, DC: Island Press.

Kotabe, H. P., Kardan, O., \& Berman, M. G. (2016a). Can the high-level semantics of a scene be preserved in the low-level visual features of that scene? A study of disorder and naturalness. In Proceedings of the 38th Annual Meeting of the Cognitive Science Society (Vol. 38, pp. 1721-1726).

Kotabe, H. P., Kardan, O., \& Berman, M. G. (2016b). The order of disorder: Deconstructing visual disorder and its effect on rule-breaking. Journal of Experimental Psychology: General, $145,1713-1727$.

Kotabe, H. P., Kardan, O., \& Berman, M. G. (2017). The nature-disorder paradox: A perceptual study on how nature is disorderly yet aesthetically preferred. Journal of Experimental Psychology: General, 146, 1126-1142.

Kuo, F. E., \& Sullivan, W. C. (2001). Environment and Crime in the Inner City: Does Vegetation Reduce Crime? Environment and Behavior, 33, 343-367.

Moisy, F. (2008). Computing a fractal dimension with Matlab: 1D, 2D and 3D Box-counting. Paris: Laboratory FAST, University Paris Sud. Paris. Retrieved from https://www.mathworks.com/matlabcentral/fileexchange/13063boxcount?focused $=5083247 \&$ tab=example

Ostwald, M. J. (2001). "Fractal Architecture": Late Twentieth Century Connections Between Architecture and Fractal Geometry. Nexus Network Journal, 3, 73-84.

Pretty, J., Peacock, J., Sellens, M., \& Griffin, M. (2005). The mental and physical health outcomes of green exercise. INTERNATIONAL JOURNAL OF ENVIRONMENTAL HEALTH RESEARCH, 15, 319-337.

Psychological Software Tools. (2012). E-Prime 2.0.

Ruskin, J., 1819-1900. (1849). The seven lamps of architecture. London: Smith, Elder, \& Co., [Spottiswoode and Shaw],.

Ryan, R. M., Weinstein, N., Bernstein, J., \& Brown, K. W. (2010). Vitalizing effects of being outdoors and in nature. Journal of Environmental Psychology, 30, 159-168.

Salingaros, N. A. (1998). A scientific basis for creating architectural forms. Journal of Architectural and Planning Research, 283-293.

Salingaros, N. A. (2003). The sensory value of ornament. Communication and Cognition, 36, 331-352.

Salingaros, N. A. (2007). A Theory of Architecture. Solingen: ISI Distributed Titles. 
Shepard, R. N. (1980). Multidimensional scaling, tree-fitting, and clustering. Science, 210, 390-398.

Shrout, P. E., \& Bolger, N. (2002). Mediation in experimental and nonexperimental studies: new procedures and recommendations. Psychological Methods, 7, 422.

Solomon, N. B. (2002). New Building Systems Mimic Nature and Return to a Biocentric Approach to Design. Architectural Record, 190, 172.

Taylor, R. P., Spehar, B., Wise, J. A., Clifford, C. W., Newell, B. R., Hagerhall, C. M., ... Martin, T. P. (2005). Perceptual and physiological responses to the visual complexity of fractal patterns. Nonlinear Dynamics Psychol. Life. Sci, 9, 89-114.

Tokkoro. (2018). Tokkoro. Retrieved March 11, 2018, from http://www.tokkoro.com/3066689-

ancient_archaeology_archeology_architecture_civilization_clouds_column_corinthianorder_cyprus_excavations_greek_heritage_historic_historical_history_kourion_landmark_ mediterranean_monument_roman.html

Ulrich, R. S. (1984). View through a window may influence recovery from surgery. Science, $224,417-419$.

Ulrich, R. S., Simons, R. F., Losito, B. D., Fiorito, E., Miles, M. A., \& Zelson, M. (1991). Stress recovery during exposure to natural and urban environments. Journal of Environmental Psychology, 11, 201-230.

Valtchanov, D., Barton, K. R., \& Ellard, C. (2010). Restorative Effects of Virtual Nature Settings. CyberPsychology, Behavior \& Social Networking, 13, 503-512.

Valtchanov, D., \& Ellard, C. G. (2015). Cognitive and affective responses to natural scenes: Effects of low level visual properties on preference, cognitive load and eye-movements. Journal of Environmental Psychology, 43, 184-195.

Vazirani, V. V. (2001). Approximation algorithms. New York: Springer.

Villani, D., \& Riva, G. (2011). Does Interactive Media Enhance the Management of Stress? Suggestions from a Controlled Study. Cyberpsychology, Behavior, and Social Networking, 15, $24-30$.

Warder, J. (2008). London - St.Paul's Cathedral - corinthian columns [Photograph]. Retrieved from https://www.panoramio.com/photo/7013775

Wilson, E. O. (1984). Biophilia. Harvard University Press.

Wilson, E. O., \& Kellert, S. R. (1995). The Biophilia Hypothesis. Island Press. 